\title{
Ministerialverwaltung und Interessengruppen - Neues und Vergessenes zu einem alten Thema
}

\author{
Marian Döhler
}

Online publiziert: 10. März 2020

(C) Der/die Autor(en) 2020

Zusammenfassung Die Bundesministerien nehmen bei der Initiierung und Formulierung politischer Vorhaben der Bundesregierung eine zentrale Rolle ein. Dies wirft immer wieder die Frage nach dem Einfluss organisierter Interessen auf. In der medialen Berichterstattung, aber auch in der wissenschaftlichen Diskussion wird die Antwort zumeist unter dem Stichwort „Lobbyismus“ gesucht. Die Ministerialverwaltung erscheint dabei überwiegend als passiver Adressat externer Einflussbestrebungen. Dieser Sichtweise soll mit Hilfe einer drei Dimensionen umfassenden Analyse entgegengetreten werden. Die Ausgangsthese lautet, dass die Ministerialverwaltung zu strategischer Interaktion befähigt ist, was zunächst auf der Makroebene der Beziehungen zwischen Staat und organisierten Interessen illustriert wird. Daran anschließend wird zweitens der Einfluss von Organisation und Arbeitsweise der Ministerialverwaltung auf die Beziehungen zu Verbänden untersucht. Die dritte Dimension schließlich gilt den Einstellungen der Ministerialverwaltung im Umgang mit organisierten Interessen. Im Ergebnis ist festzustellen, dass die Interaktionen zwischen beiden Seiten deutlich über die Automatismen der Interessendurchsetzung hinausgehen, wie sie im Lobbyismus-Konzept unterstellt werden.

Eine frühere Fassung dieses Aufsatzes wurde auf der Tagung der DVPW-Sektion „Policy-Analyse und Verwaltungswissenschaft“ am 19. und 20.09.2019 an der Deutschen Universität für Verwaltungswissenschaften Speyer präsentiert. Für hilfreiche Kommentare und Verbesserungsvorschläge danke ich den Diskussionsteilnehmern, insbesondere Stephan Grohs, den ZPol-Gutachtern sowie Jasmin Beneke, Florian Groß, Fabian Jakubowitz und Christina Lichtmannegger.

M. Döhler $(\triangle)$

Institut für Politikwissenschaft, Gottfried Wilhelm Leibniz Universität Hannover,

Schneiderberg 50, 30167 Hannover, Deutschland

E-Mail: m.doehler@ipw.uni-hannover.de 


\title{
Ministerial bureaucracy and interest groups - New and forgotten perspectives on an old topic
}

\begin{abstract}
German Federal Ministries play a central role in the initiation and formulation of political initiatives by the Federal Government. This repeatedly raises the question of interest group influence. In media reporting, but also scientific discussions, the answer is usually sought under the keyword "lobbyism", in which the ministerial bureaucracy appears to be largely a passive recipient of external influence. This view is challenged by applying a three-dimensional analysis. The initial hypothesis is that the ministerial bureaucracy is capable of strategic interaction, which is first illustrated at the macro level of relations between the state and organized interests. Second, the effect of organization and procedures of the ministerial bureaucracy on the relationship with interest groups is examined. Finally, the attitudes of ministerial bureaucrats in dealing with organized interests are reconstructed by using data from an online survey. The result is that the interactions between the two sides go far beyond the one-way automatism suggested in the lobbying concept.
\end{abstract}

\section{Problemstellung}

Das Verhältnis zwischen Staat und Verbänden wird seit einiger Zeit überwiegend unter dem Stichwort „Lobbyismus“ behandelt. Dabei sind zwei thematische Stoßrichtungen erkennbar. Auf der einen Seite stehen die politischen Einflussbestrebungen großer Verbände sowie die Ausdifferenzierung der Interessengruppenlandschaft im Vordergrund. Hier wird betont, dass eine wachsende Zahl von Verbänden ${ }^{1}$ oder Lobbyisten nicht unbesehen mit mehr Einfluss gleichgesetzt werden darf, weil das System organisierter Interessen ,vielgliedriger“ (Schmidt 2011, S. 118; Kohler-Koch 2016; Sack und Strünck 2016) geworden und ,die Bindekraft traditioneller Verbände“ (Lösche 2007, S. 117) gesunken sei. Auf der anderen Seite wird in einem eher journalistisch/zivilgesellschaftlich inspirierten Diskurs (so etwa Leif und Speth 2006; Göhler et al. 2012) davon ausgegangen, dass staatliche Instanzen den Forderungen ressourcenstarker Verbände nur wenig entgegenzusetzen haben und gemutmaßt, dass ,hinter verschlossenen Türen bestimmte Interessen zum Zuge kommen und andere nicht" (Niederhafner und Speth 2004, S. 30). Gemeinsam ist beiden Literatursträngen, dass sie sich von typologischen Ansätzen, die Lobbyismus als Merkmal eines pluralistischen Systems der Interessenvermittlung konzipieren, entfernt haben und eine Perspektive einnehmen, die Verbände und ihre Aktivitäten in den Vordergrund rücken. Während im Hauptstrang typologischer Ansätze, der Korporatismusdebatte, noch die wechselseitige Einflussnahme oder kooperative Beziehungen zwischen staatlichen und privaten Akteuren im Vordergrund standen (vgl. zusammenfassend Jochem und Siegel 2003), wird mit dem Lobbyismus-Framing eine generelle, allerdings einseitige Antwort auf die Frage gegeben, welche Me-

\footnotetext{
1 Die Zahl, der beim Bundestag registrierten Verbände (https://www.bundestag.de/parlament/lobbyliste/) hat sich seit 1990 von 1501 auf 2339 im Jahr 2020 erhöht (Stand: 2/2020), was einer Zunahme von rund $36 \%$ entspricht.
} 
chanismen das Verhältnis zwischen Staat und Verbänden bestimmen, insbesondere welche Interessen letztlich die Oberhand gewinnen (neuere Ausnahme: Schiffers 2019).

An diesem Punkt setzen die nachfolgenden Überlegungen an. Die von Wilhelm Hennis formulierte und empirisch verschiedentlich bestätigte These (vgl. Weber 1981, S. 290-292; Mayer und Naji 2000; Sebaldt und Straßner 2004, S. 153), wonach ,nicht das Parlament, sondern Regierung und Verwaltung der entscheidende Adressat des Verbandseinflusses sind“ (Hennis 1961, S. 25), wird heute so verstanden, als könne man daraus bereits auf ein Ergebnis schließen. Der zutreffenden Feststellung, dass die Bundesministerien, in denen das Gros der Gesetzesformulierung stattfindet, von Interessengruppen als „Lobby-Adressaten“ (Wehrmann 2007, S. 43; Lösche 2007, S. 66-70; Baruth und Schnapp 2015) wahrgenommen werden, droht in Verbindung mit einem analytisch uninteressierten Lobbyismus-Konzept eine perspektivische Verengung, in weder eine Unterscheidung zwischen Einflussversuchen und erfolgreicher Einflussnahme stattfindet, noch die Variabilität der Einflussrichtung zwischen Staat und Verbänden bedacht wird. Im Ergebnis verstärkt die aktuelle „Lobbyismushysterie“ (Czada 2015, S. 12) im Zusammenspiel mit der Berichterstattung über externe Beratung sowie über das Gesetzgebungsoutsourcing den Eindruck, dass sich Politik und Ministerialverwaltung heillos in einem Netz aus durchsetzungsfähigen Partikularinteressen verstrickt haben.

Dem soll hier die Hypothese entgegengehalten und empirisch untermauert werden, dass die Ministerialverwaltung nicht nur Adressat externer Einflussversuche ist, sondern mehr Arena der Politikformulierung mit eigener Befähigung zu strategischer Interaktion. Dies schließt an vielfach vergessene Überlegungen aus der Korporatismus-Debatte an, in der die Indienstnahme privater Akteure für staatliche Steuerungsziele noch ausführlich thematisiert wurde. Die heute für den Erfolg von Interessengruppen gern in Anschlag gebrachten Faktoren, wie Kontakthäufigkeiten, Anzahl von Lobbyisten, Verbandsressourcen oder Drohungen mit Abwanderung oder Arbeitsplatzabbau bleiben aus dieser Perspektive unvollständig, wenn nicht gar einseitig. Akzeptiert man hingegen, dass die Ministerialverwaltung eine aktive Rolle in der politischen Willensbildung spielt und nicht nur eine Echokammer für Verbandsforderungen darstellt, dann gelangen Faktoren in den Blick, die auf drei Dimensionen angesiedelt sind.

In Abschnitt zwei steht zunächst die Makroebene des Verhältnisses zwischen Ministerialverwaltung und ihrer Umwelt im Mittelpunkt. Dieser Analyseschritt behandelt kooperative Formen der Interessenvermittlung, die sich jenseits von LobbyAktivitäten herausgebildet haben. Die zweite Dimension (Abschn. 3) umfasst Arbeitsweise und Binnenorganisation der Bundesministerien, die zwar durchaus Gegenstand der Forschung sind, aber selten die Frage thematisieren, welcher Einfluss von diesen Faktoren für das Verhältnis zu Verbänden ausgeht. Die hier eingenommene Perspektive wirft ein neues Licht auf diese Analysedimension. In diesem Kontext wird auch die Beteiligung Externer an der Gesetzesformulierung rekapituliert. Im vierten Abschnitt wird schließlich auf die Einstellungen der Ministerialverwaltung im Umgang mit organisierten Interessen eingegangen und die drei Analysedimension zu einem übergreifenden Wirkungsmodell zusammengeführt. 


\section{Verbände in der bundesdeutschen Verhandlungsdemokratie}

Erkenntnisfortschritte lassen sich nicht nur mit neuen Konzepten und Theorien erzielen, sondern auch durch die Rückbesinnung auf ältere Fachdiskurse. Ein kurzes Gedächtnis liegt bereits der verbreiteten Neigung zugrunde, Interessengruppen mit Lobbyismus gleichzusetzen, der dann häufig als gemeinwohlabträgliche Verfolgung von Sonderinteressen betrachtet wird. Diese Sichtweise lässt einen Diskussionsstand außer Acht, in dem Verbänden noch ein deutlich breiteres Funktionsprofil zugerechnet wurde. Diese prozessorientierte Perspektive sieht die Interessenaggregation als Ausgangspunkt, bei der eine Vielzahl einzelner und nicht selten widersprüchlicher Mitgliederinteressen zu politisch handhabbaren Positionen und Forderungen transformiert werden. Erst diese Bündelungsleistung macht die anschließende Interessenartikulation in einer Weise möglich, die mit dem politischen Prozess kommensurabel ist. Drittens folgt die Interessenselektion, verstanden als jene ,Wirkungen der Verbände in ihrer Gesamtheit auf das politische System“ (Weber 1981, S. 389) ausüben. Summarisch wird dieser dreistufige Prozess zumeist als Interessendurchsetzung bezeichnet. Nicht ohne Grund verwenden manche Autoren anstelle dessen den Begriff der Interessenvermittlung (so etwa Lehmbruch 1987; Streeck 1994, S. 12). Damit wird Verbänden kein „,fertiges“, aus der Summe der Mitgliederpräferenzen geronnenes Interesse unterstellt, welches dann nur noch der Durchsetzung bedarf. Vielmehr signalisiert der Begriff der Interessenvermittlung, dass Positionen und Forderungen nicht bereits im Moment der Aggregation feststehen, sondern erst durch die Interaktion mit politischen Instanzen, also auch den Bundesministerien, ihre endgültige Form erhalten.

Dies ist wichtig für das Verständnis des staatlichen bzw. ministeriellen Handlungskalküls im Umgang mit organisierten Interessen. Verbände portionieren das Gewirr gesellschaftlicher Interessen für die Weiterbearbeitung im politischen Prozess und können aufgrund dieser Aggregationsleistung eher Kompromisspositionen vertreten als ihre Mitglieder. Während die Aufmerksamkeit heute primär den Einflussbestrebungen von Verbänden auf den politischen Prozess gilt, stand im Zuge der Korporatismus-Diskussion noch die gegenläufige Richtung im Vordergrund, nämlich Interessengruppen für staatliche Steuerungsziele einzubinden und deren Kooperationsbereitschaft durch politische Tauschgeschäfte zu stimulieren (vgl. Czada 1994). Die Mehrzahl der Autoren misst dem Korporatismus-Konzept nach dem Scheitern der wichtigsten institutionellen Ausprägungen, der Konzertierten Aktion beim Wirtschaftsminister (1967-1976) sowie dem Bündnis für Arbeit (1998-2002), keine prägende Bedeutung mehr für die politische Praxis der Bundesrepublik bei (vgl. Streeck 2003; Loer und Voelzkow 2013). Das sollte allerdings weder ein Grund sein, die aus diesem Diskurs gewonnenen Lehren ad acta zu legen, noch die Dauerhaftigkeit kooperativer Staat-Verbände-Beziehungen zu ignorieren. Denn die bundesdeutsche Politik ist noch immer von einer Vielzahl konsultativer Gremien und Verfahren geprägt, die mittlerweile unter dem Begriff der Verhandlungsdemokratie subsummiert werden (vgl. Lehmbruch 2003; Czada 2015). Dazu gehören die Kommissionen und Räte der Regierung Schröder (vgl. Heinze 2002; Siefken 2007), die bis heute anhaltende Kette von Energie-, Bildungs-, Digital- oder Integrations-Gipfeln (vgl. Krick 2010; Bundestag 2019b), die Kohlekommission oder das mit einer eigenen Behörde 
versehene Endlagersuchverfahren. In der Regel handelt es sich um Initiativen, die von der Bundesregierung oder einzelnen Ministerien ausgehen. Es wäre sicher überzogen, dafür den Korporatismus-Begriff zu bemühen. Aber die urwüchsige Wiederkehr derartiger Kooperationsformen lässt auf eine verhandlungsdemokratische Grundorientierung in Politik und Verwaltung schließen, die auch nach dem Ende einer zentral organisierten „Makrokonzertierung“ (Jochem und Siegel 2003, S. 16) ihre strategieprägende Wirkung nicht verloren hat.

In einem eher öffentlichkeitsabgewandten Modus findet die finanzielle Unterstützung von Verbänden statt, die sich seit den 1950er Jahren etabliert hat (vgl. dazu Wambach 1971, S. 138-142) und in jenen Politikfeldern besonders ausgeprägt ist, in denen Interessengruppen als Verbündete von Ministerien agieren, etwa im Umwelt- und Naturschutz, der Agrarpolitik, der Entwicklungszusammenarbeit, der Familienpolitik oder der Sportförderung. Diese und andere Beziehungsmuster lassen sich nach Mission, Strategie und Struktur unterscheiden (vgl. Fraussen 2014, S. 409). Bundesministerien agieren missionsgetrieben, wenn sie Verbände als verlängerten Arm für die eigenen Ziele in Dienst nehmen. Beispiele sind die Umweltund Naturschutzverbände wie der WWF, der BUND, der NABU oder die Deutsche Umwelthilfe, die als ,externe Interessenträger“ (Bundestag 2019a) sechs- bis siebenstellige Fördermittel zur Durchführung von Kampagnen oder Projekten erhalten. Auch wenn die finanzielle Unterstützung meist befristet gewährt wird, deuten die in den Haushaltsplänen der Ressorts enthaltenen Titel (684-686) darauf hin, dass es sich mittlerweile um eine ministerielle Daueraufgabe handelt. Als strategiegetrieben lassen sich Maßnahmen wie die „Förderung der Selbstorganisation von Migrantinnen und Migranten“ begreifen, die das „Engagement für Demokratie und für Vielfalt“" (Bundestag 2018, S. 11) unterstützen soll. Beide Motive dürften bei der dauerhaften und mit Zielvereinbarungen versehenen Förderung der Sportfachverbände durch das Bundesministerium des Inneren (BMI) zusammenfallen (Bundesrechnungshof 2014). Strukturgetrieben sind schließlich Bestrebungen, die Organisation der Verbände bzw. deren Machtverteilung untereinander im Sinne staatlicher Ziele $\mathrm{zu}$ beeinflussen.

Deutlich erkennbar ist das an der ministeriellen Vorliebe für Repräsentationsmonopole (vgl. Offe 1981), die sich bereits in der Weimarer Republik abzeichnete, als das Reichsarbeitsministerium den Spitzenverbänden der Wohlfahrtspflege eine privilegierte Position einräumte (Backhaus-Maul und Olk 1994, S. 104 f.). Anlässlich der Fusion dreier Verbände zu einem ,Spitzenverband der landwirtschaftlichen Sozialversicherung“ stellte ein Pressekommentar fest: „Die Bundesregierung scheint das Wort ,Spitzenverband“ zu lieben“ (BDI 2008). In der Tat zählen die „,kommunalen Spitzenverbände“, die „Spitzenverbände der freien Wirtschaft““ oder die „Spitzenverbände der freien Wohlfahrtspflege“ zu den bevorzugten Ansprechpartnern von Politik und Verwaltung. In gesetzlichen Regelungen ist daher häufig von ,beteiligten Kreisen“2 die Rede oder die „Spitzenverbände der Kreditwirtschaft“ (§ 23 Abs. 2 Kreditwesengesetz), die „Spitzenverbände der zuständigen Gewerkschaften“ ( $\$ 94$ Bundesbeamtengesetz) bzw. die „Spitzenverbände der Unternehmen“ ( $\$ 308$ b Ver-

\footnotetext{
${ }^{2}$ So etwa in $\S 51$ Bundes-Immissionsschutzgesetz; § 58 Abs. 1 Bundes-Naturschutzgesetz; §§ 5, 11 Tarifvertragsgesetz; § 17 Abs. 1 Chemikaliengesetz.
} 
sicherungsaufsichtsgesetz) werden explizit als zu Beteiligende benannt (vgl. auch Weber 1981, S. 198-200). Daraus lässt sich schließen, dass die Ministerialverwaltung bei der Unterstützung von Spitzen- und Dachverbänden das Ziel verfolgt, sich auf einen Ansprechpartner stützen zu können, um so den Willensbildungsprozess zu vereinfachen bzw. zu beschleunigen. Dabei spielt die Verpflichtungsfähigkeit, also die Sicherung der verbandsinternen Folgebereitschaft gegenüber getroffenen Absprachen, eine wesentliche Rolle und macht plausibel, warum neben Finanzhilfen und privilegierten Zugängen auch die Initiierung von Verbandsfusionen in das Strategierepertoire der Ministerialverwaltung gehört.

Unter tätiger Mithilfe des seinerzeit für den Umweltschutz zuständigen BMI erfolgte 1972 die Gründung des Bundesverbandes Bürgerinitiativen Umweltschutz mit dem Ziel, die ,amorphe[n] Organisationsstrukturen“ (Müller 1986, S. 119) der Bürgerinitiativen zu konsolidieren, um deren Einbindung in politische Entscheidungsprozesse zu ermöglichen. Ein anderes Beispiel ist der 1991 gegründete Bundesverband Erneuerbare Energien, der die zersplitterte Verbändelandschaft in diesem Bereich zu einer handlungsfähigen Einheit zusammenschließen sollte (vgl. Stefes 2014, S. 62). Mit dem Deutschen Olympischen Sportbund, der 2006 aus der Verschmelzung des Nationalen Olympischen Komitees und des Deutschen Sportbundes hervorging, hat sich das Innenministerium eine Art „Ergänzungsbürokratie“ (Wambach 1971, S. 131) für seine Spitzensportambitionen geschaffen (vgl. Braun und Reymann 2013). Auch die Gründung der Verbraucherzentrale Bundesverband e.V. als Dachverband im Jahr 2001 geht auf eine ministerielle Initiative zurück, die das Bundesministerium für Wirtschaft (BMWi) teilweise gegen die Verbraucherverbände umsetzen konnte (vgl. Tagesspiegel 1999). Ebenfalls gegen den Widerstand der sieben Kassenarten gelang dem Bundesgesundheitsministerium (BMG) 2008 die Errichtung eines „Spitzenverbandes Bund der Krankenkassen“ (vgl. Paquet 2009), womit ein langer Prozess ministeriell betriebener Korporatisierung seinen Abschluss fand (vgl. dazu ausführlich: Döhler und Manow 1992, 1997). Gelingen konnte das u. a. deshalb, weil die Kassenverbände als Körperschaften des öffentlichen Rechts konstituiert sind, deren Aufgaben und Organisation im Sozialgesetzbuch geregelt sind. Die Strategie, Verbände für staatliche Steuerungsziele zu instrumentalisieren, ist demzufolge an Gelegenheitsstrukturen gebunden, die nicht in allen Politikfeldern vorhanden sind. Ablesbar ist das u. a. an den mehrfach gescheiterten Versuchen des Innenministeriums, die islamischen Verbände zum Zusammenschluss in einen Dachverband zu bewegen (vgl. Blätte 2010).

Struktur- bzw. strategiegetriebene Aktivitäten zielen häufig auf eine Kräfteverschiebung im jeweiligen Politikfeld, die im staatlichen Interesse liegt. Dies war Kern der bereits erwähnten Korporatisierungsstrategie des BMG, die die Kassenverbände durch eine Zentralisierung gegenüber den Leistungserbringern zu stärken beabsichtigte. Explizit als Gegengewicht zum Deutschen Atomforum erfolgte 1989 mit einer Anschubfinanzierung des BMWi die Gründung des ,Forums für Zukunftsenergien“ (vgl. Czada 1991, S. 161-162). Im Rahmen des Programms „Demokratie leben!“ betreibt das Familienministerium seit einigen Jahren systematisches „,apacity building " für zivilgesellschaftliche Akteure. Die hierfür eingerichtete Förderlinie „Strukturentwicklung zum bundeszentralen Träger" soll NGOs mit finanzieller Unterstützung, mitunter auch weisungsähnlichen Vorschlägen (im Ministerialjargon 


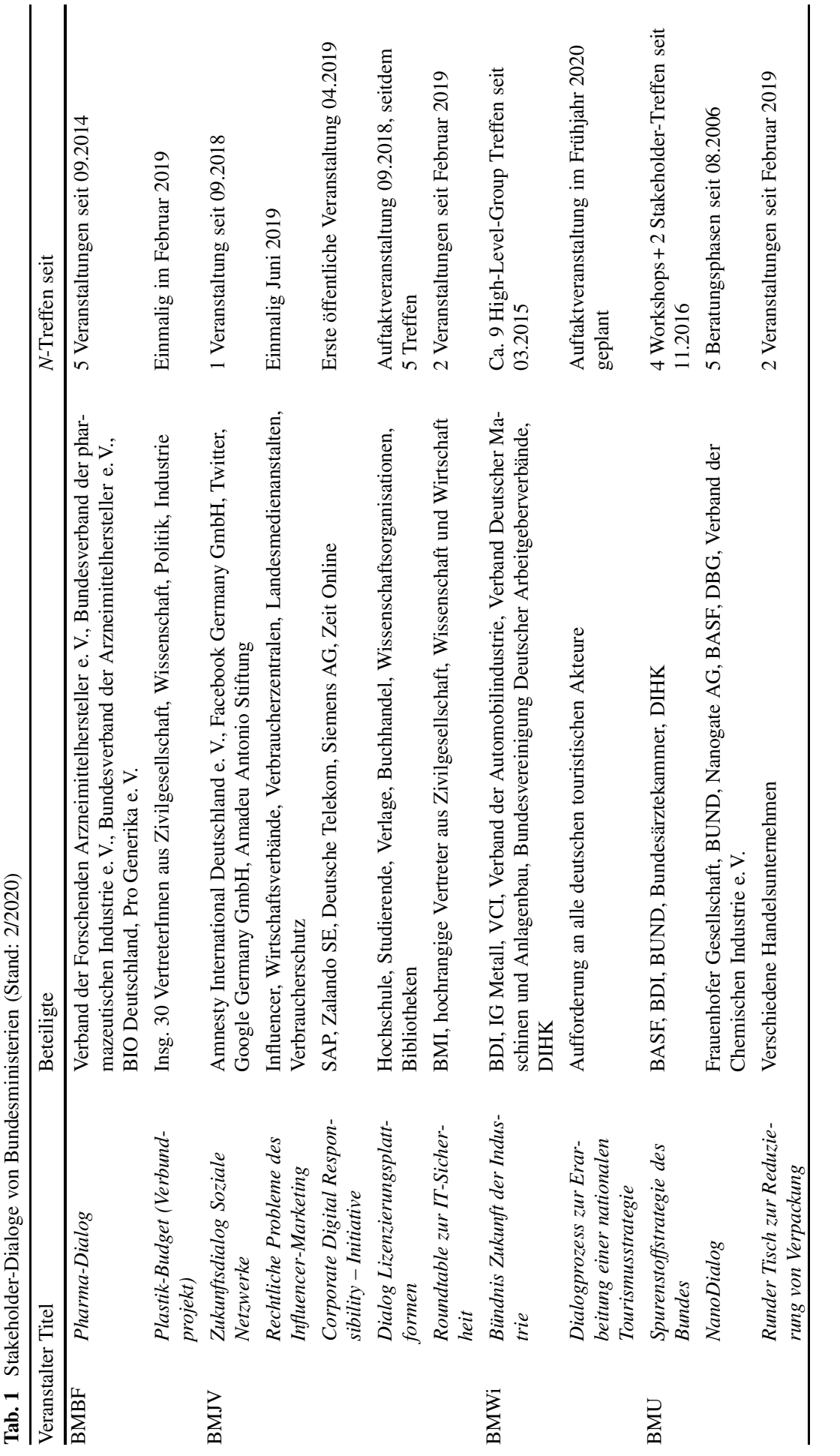




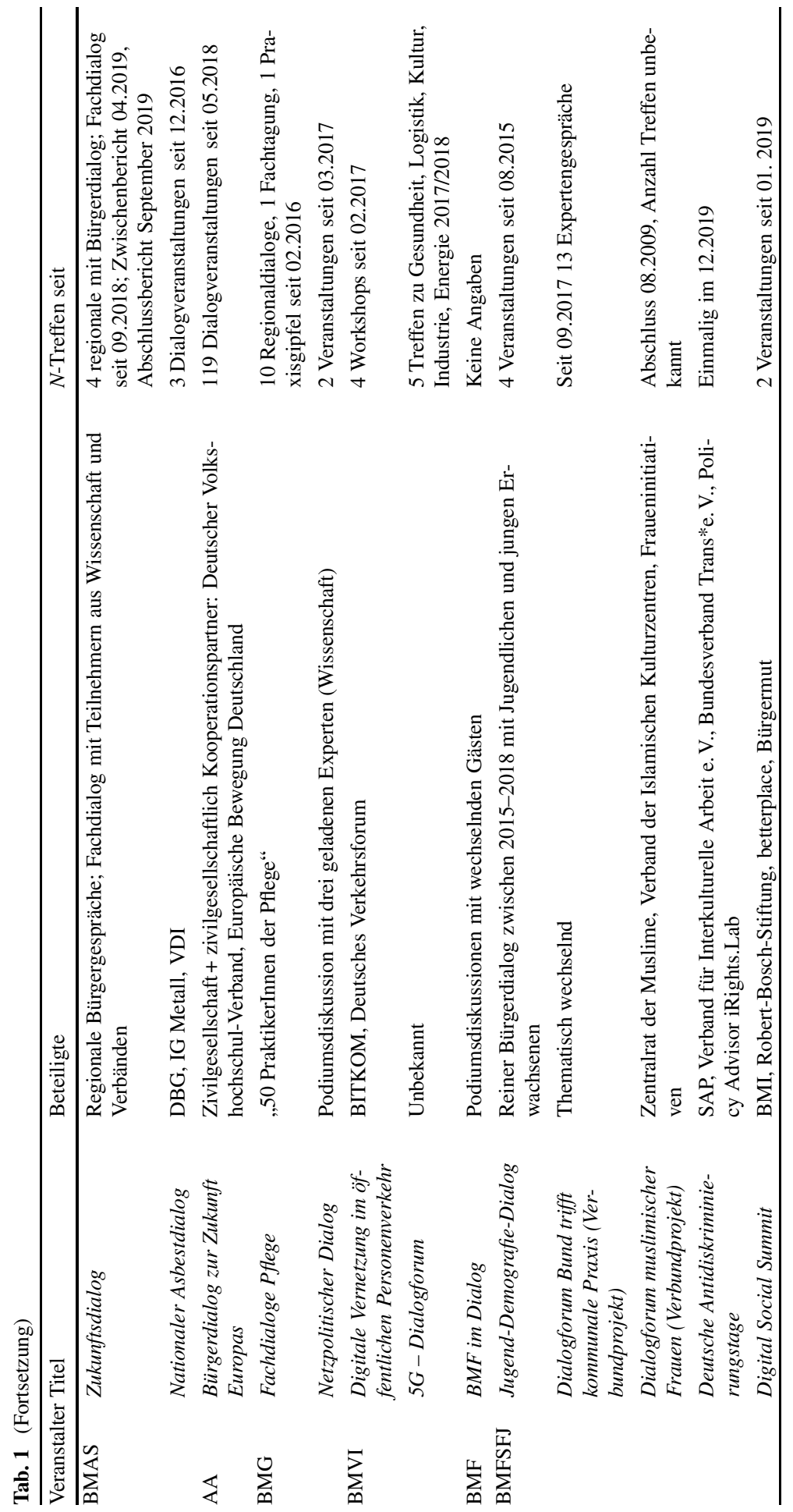




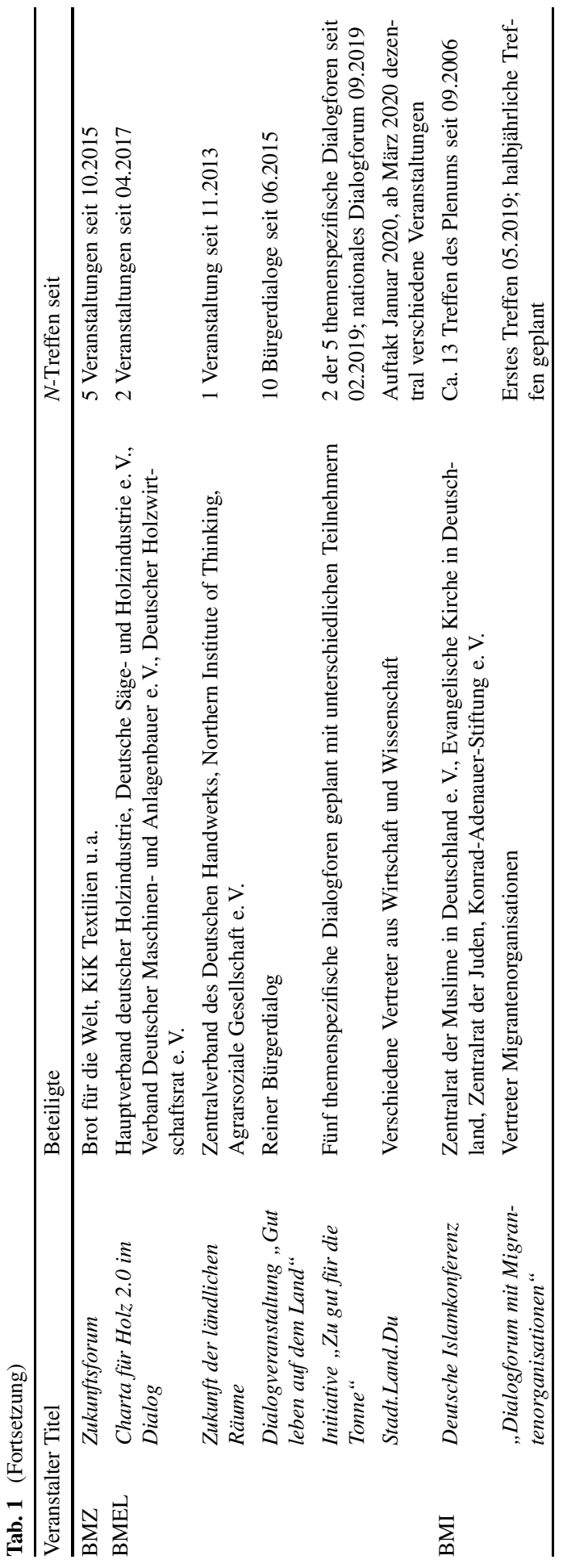


heißt das „Fachberatung“) organisatorisch in die Lage versetzen, als Advokaten für ministerielle Programme zu agieren (vgl. Heinze und König 2019).

Ministerielle Gestaltungsinitiativen im Umgang mit organisierten Interessen bzw. zivilgesellschaftlichen Akteuren variieren also einerseits politikfeldspezifisch. Andererseits können generelle Entwicklungen wirksam werden, deren Ursachen im Wandel der Verbändelandschaft zu vermuten sind. So lässt sich der Umstand erklären, dass bis auf das Verteidigungsministerium mittlerweile alle Ressorts auf sog. Stakeholder-Dialoge zurückgreifen, einem aus der Privatwirtschaft entlehnten, zwischen Imagepflege und Konsensbildung oszillierenden Veranstaltungsformat. Die in Tab. 1 aufgelisteten Dialoge vermitteln zwar von der Thematik, den Teilnehmern sowie dem Veranstaltungsturnus her ein buntes Bild. Dass sich dieses Format aber seit Mitte der 2000er auf breiter Fläche durchgesetzt hat, deutet auf einen ,inverse[n] Lobbyismus“" (Urban 2010, S. 4) hin, bei dem es der Ministerialverwaltung nicht nur um die Entwicklung gemeinsamer Problemdefinitionen und Lösungsvorschläge geht, sondern mitunter auch deutlich ambitioniertere Ziele verfolgt werden. Der mittlerweile beendete „Forschungsdialog Futur“ sollte u. a. für eine „,bessere Legitimation der Förderpolitik des BMBF“ (Adam 2004, S. 86) sorgen. Mit dem Dialogprozess „Arbeiten 4.0“, der zwischen 2015 und 2016 stattfand, wollte sich das Bundesministerium für Arbeit und Sozialordnung gar als „Diskursgestalter“ etablieren, dem die „Meinungsführerschaft“"3 in der Bewältigung technologischer Umbrüche der Arbeitswelt zufällt. Wenn es für die Ministerialverwaltung infolge der wachsenden Fragmentierung der Verbändelandschaft sowie des Wachstums eher schwach organisierter NGOs schwieriger wird, verpflichtungsfähige Kooperationspartner zu finden, dann könnten die Stakeholder-Dialoge als anspruchsärmere Variante der Konsensund Legitimationsbeschaffung fungieren. Jenseits dieser Überlegungen verdeutlichen die hier aufgeführten Beispiele, dass verhandlungsdemokratische Verfahren in erklecklicher Zahl existieren, die nicht vom Lobbyismus-Framing erfasst werden.

\section{Organisation und Arbeitsweise der Ministerialverwaltung in der Interessenvermittlung}

Auch die zweite Analysedimension kann auf der vorhandenen Literatur aufbauen, allerdings unter einer veränderten Perspektive. Lange Zeit herrschte die Annahme vor, dass ganze Ressorts durch Interessengruppen kolonisiert werden. Diese Befürchtung hat sowohl durch die Rekrutierung von Leitungspersonal aus der Verbandszene (für Beispiele vgl. Wambach 1971, S. 125-131; Weber 1981, S. 260-261), wie auch durch die Verbands-Affinität einzelner Ministerien immer wieder Nahrung erhalten. In den 1950er Jahren galten das Bundesministerium für Vertriebene, das Bundesministerium für Atomfragen sowie - bis in die jüngere Vergangenheit - das Bundesministerium für Ernährung und Landwirtschaft als typische „Klientelministerien“. Geringer sei das „Ausmaß der Interessenprägung“ bei den sog. klassischen Ressorts

\footnotetext{
3 Beide Zitate stammen vom seinerzeit zuständigen Unterabteilungsleiter des BMAS während eines Workshops zum Thema „Projektgruppe Regierungs- und Verwaltungsreform nach 40 Jahren - was kann Wissenschaft und Praxis heute lernen“, Hertie School of Governance am 17.06.2017.
} 
(Inneres, Äußeres, Finanzen, Verteidigung und Justiz), weil deren Aufgaben „so allgemein [seien], dass sie nicht das wesensmäßige Interesse einer gesellschaftlichen Gruppe ausmachen“ (Steinberg 1979, S. 250. Hervorhebung i.O. MD). Dass dem ,policy portfolio“, also dem Aufgabenmix, große Bedeutung für die Beziehungen zu Verbänden beigemessen wird, ist plausibel, weil sich darin auch die Anzahl und das Gewicht konkurrierender Interessen wiederspiegelt, die ministeriumsintern ausbalanciert werden müssen. Inwieweit die wachsende Fragmentierung der Verbändelandschaft die Chancen für Tauschgeschäfte und damit den Umgang der Ministerialverwaltung mit Interessengruppen tangiert, ist eine weitgehend unbeachtete Frage, die weiter unten noch einmal aufgegriffen wird.

Für alle Ressorts gleichermaßen gültig sind die formalen Regeln im Umgang mit Interessengruppen. Zentrale Bedeutung kommt der Gemeinsamen Geschäftsordnung der Bundesministerien (GGO) $\mathrm{zu}_{5}$ die - früher noch an zwei Stellen - ,den Zugang zum Machthaber“ (Hennis 1961, S. 27) regelt. Die Informations- und Anhörungsmöglichkeiten im Stadium eines Referentenentwurfs ( $\$ 23$ GGO II [a. F.]) war durch die explizite Begrenzung des „Verkehrs“ auf „Zentral- und Gesamtverbände“ ( 77 GGO I [a. F.]) komplettiert. Damit sollte einer Überlastung durch die Kontaktaufnahme regionaler Verbandsorganisationen entgegengewirkt werden. Auch in der heute gültigen Fassung der GGO ist ,eine rechtzeitige Beteiligung von Zentral- und Gesamtverbänden sowie von Fachkreisen, die auf Bundesebene bestehen“ ( $\$ 47$ Abs. 3 GGO), vorgesehen. Wie das im Einzelnen zu geschehen habe und welchen Verbänden eine „rechtzeitige Beteiligung“ ermöglicht werden soll, bleibt „,dem Ermessen des federführenden Bundesministeriums überlassen“ ( $\$ 47$ Abs. 3 GGO). ${ }^{4}$ Darin scheint der ursprüngliche Zweck dieser Regelung auf, die weder einen Anspruch auf Gehör begründen sollte noch als Einladung gemeint war, sondern abwehrenden Charakter besaß (näheres bei Hennis 1961, S. 28). Diese Stoßrichtung wird auch in einer mittlerweile entfallenen Regelung deutlich, die das Störpotential partikularer Interessenverfolgung auf der Ebene der Ressortkoordination zu reduzieren suchte. Dort hieß es: „Im Übrigen ist darauf zu achten, daß mit Vertretern der Fachkreise oder Verbände nicht in einer Weise Fühlung genommen wird, die dem Kabinett die Entscheidung erschwert" (§ 24 Abs. 3 GGO II [a.F.]). ${ }^{5}$ Dass diese Problematik im ministeriellen Bewusstsein noch immer präsent ist, kann an der im Jahr 2000 eingefügten Vorgabe für Kabinettvorlagen abgelesen werden, der zufolge „das Ergebnis einer Verbändebeteiligung, insbesondere die Darstellung wesentlicher Anregungen, denen nicht entsprochen werden soll“" ( 22 Abs. 1 GGO), aufzuführen ist. Es wäre also zumindest einseitig, die GGO nur als „Magna Charta des Verbandseinflusses“ (Weber 1981, S. 195) zu betrachten und ihre Abwehrintentionen auszublenden (ähnlich Sebaldt und Straßner 2004, S. 144).

\footnotetext{
${ }^{4}$ Auch für die bereits erwähnten gesetzlichen Beteiligungsmöglichkeiten wird festgestellt, dass dies ,kein scharfes Schwert für die Spitzenorganisationen“ sei „bestimmte [gesetzliche] Regelungen zu erzwingen oder zu verhindern" (Hebeler 2018, S. 1369. Hervorhebung i.O. [MD]).

${ }^{5}$ In der Ursprungsfassung der „Gemeinsamen Geschäftsordnung der Reichsministerien“ aus dem Jahr 1926 war diese Regelung noch schärfer formuliert: „Bevor Ministerien einig sind, darf nicht mit Fachkreisen in einer Weise Fühlung genommen werden, die eine den Forderungen der Fachkreise unerwünschte Entscheidung in irgendeiner Weise erschwert" (\$ 27 GGO II [a. F.]).
} 


\subsection{Organisation, Information und Kontrolle}

Auch wenn die innere Organisation und die Arbeitsweise der Ministerialverwaltung kaum unter dem Aspekt der Einflusschancen von Interessengruppen thematisiert werden, lässt sich die Dominanz der negativen Koordination zwischen und innerhalb von Ministerien (vgl. Hustedt und Veit 2014) so verstehen, dass Lobby-Bestrebungen immer erst konkurrierende Interessen und davon ausgelöste „Mitzeichnungskriege“ (Murswieck, zit. nach Hustedt 2013, S. 115) überwinden müssen. Eher am Rande findet sich der Hinweis, dass der dezentrale Aufbau der Bundesministerien den Referaten eine relative Autonomie erlaube, die sie gegen externen Druck unempfindlich mache (vgl. Mayntz und Scharpf 1975, S. 71). Andererseits wird argumentiert, dass „Strukturen [und] Arbeitsprozesse in den Ministerien ... von Lobbyisten genutzt werden“ (Baruth und Schnapp 2015, S. 252) könnten. Die Herausbildung privilegierter Außenkontakte ließe sich auch organisationstheoretisch begründen, da eine divisionale Struktur in der Regel die Kontrolle über die dezentralen Einheiten schwächt (vgl. Döhler 2017).

Im Kern laufen derartige Wirkungshypothesen auf den Bedarf bzw. die mutmaßliche Abhängigkeit von externen Informationen hinaus (vgl. Steinberg 1979, S. 245; Weber 1981, S. 197; Mai 2018, S. 13-15). Verbändekontakte dienen demnach - wie es in $\S 23$ Abs. 1 der GGO I [a. F.] hieß - ,zur Beschaffung von Unterlagen für die Vorbereitung von Gesetzen“, die auf anderem Wege nicht erhältlich sind. Während die formale Verbändebeteiligung zu einem Zeitpunkt stattfindet, an dem der Referentenentwurf bereits inhaltliche Weichen gestellt hat, können informelle Kontakte auf die vorgelagerte Problemwahrnehmung und die Auswahl von Lösungsalternativen einwirken. Mayntz betrachtet Konsultation als Versuch der ,gedanklichen Vorwegnahme von Widerständen“ (Mayntz 1978, S. 209) durch die Ministerialverwaltung. Die Mehrzahl der Autoren betont hingegen die Einspeisung interessengefärbter Informationen oder die Initiierung politischer Vorhaben (vgl. u. a. Sebaldt und Straßner 2004, S. 156-162; Lösche 2007, S. 67; Baruth und Schnapp 2015, S. 252), ohne allerdings die Frage zu klären, welche Wirkung derartige Kontakte letztendlich erzielen.

Hier ist eine argumentative Schwachstelle genereller Natur zu beobachten. Dass Verbände politische Themen auf die Agenda heben, oder umgekehrt, Vorhaben verzögern, verwässern oder gar blockieren können, ist unbestritten. Ob es sich dabei gemessen an der Gesamtzahl ministerieller Vorgänge aber um viele oder um wenige Fälle handelt und worin die Ergebnisse bestehen, wird kaum thematisiert, und dies vermutlich aus gutem Grund. Denn die Ubiquität informeller Kontakte lässt sich von ihrem Umfang, den Inhalten und Wirkungen her empirisch kaum so präzise erfassen, dass die Durchsetzungsfähigkeit organisierter Interessen generell beurteilt werden könnte. Dies gemahnt zur Vorsicht gegenüber all jenen Stimmen, die bereits Aktivitäten von Verbänden mit Lobby-Erfolgen gleichsetzen.

Auch das Argument von der Informationsabhängigkeit ist nicht nur unspezifisch, weil es weder nach Art und Zweck der Informationen differenziert, die ja auch der Durchsetzung ministerieller Interessen dienen können, sondern übergeht zudem all jene Informationsquellen, die der Ministerialverwaltung routinemäßig zur Verfügung stehen. Dazu zählen das Dienstwissen erfahrener Mitarbeiter/innen (vgl. 
Pfeiffer und Faller 1997, S. 23, 35), ,das eigene Archiv“, welches „,nachfolgende[n] Generationen von Beamten und Politikern“ auch ,weiter zurückliegende Vorgänge zu demselben ,Aktenzeichen“" (Mai 2016, S. 142) mit Informationen und Bewertungen erschließt, sowie nicht zuletzt der nachgeordnete „Geschäftsbereich“, dessen meist hoch spezialisiertes Personal vielfach routinemäßig in die Politikformulierung eingebunden ist (vgl. dazu umfassend: Döhler 2007; Bach 2012). Selbst wenn informelle Konsultationen vielschichtig wirken und es ihnen an Transparenz mangelt, sind Zweifel an der Interpretation angebracht, dass sich Interessengruppen auf diesem Wege ausschließlich oder auch nur mehrheitlich durchsetzen.

Mitunter werden die Leitungsebene bzw. deren Beziehungen zur Arbeitsebene als organisatorischer Schwachpunkt betrachtet, der von Interessengruppen genutzt werden könne. So lautet eine These, dass Verbände sich primär , dort für die Verwaltung [interessieren], wo diese in einem Bereich der relativen Autonomie gegenüber der politischen Führung handeln kann" (Weber 1981, S. 282). Durchsetzungschancen gegenüber einem Ministerium lassen sich demzufolge bei Vorhaben vermuten, die entweder im Windschatten der politischen Aufmerksamkeit liegen oder mit den Interessen der Leitung kompatibel sind. Letzteres wird von Insidern bestätigt (Müller 2013, S. 57-58; Mai 2016, S. 140-142), ist aber insofern trivial, als es gar keiner Durchsetzung von Interessen bedarf, wenn die Ressortleitung per se mit externen Wünschen übereinstimmt. Gegen die verbreitete Vorstellung, dass der direkte Kontakt zur Leitung bereits eine erfolgreiche Einflussnahme bedeutet, spricht die gängige Praxis, dass Gespräche von Minister/innen mit Interessenvertretern von der Arbeitsebene vorbereitet, begleitet und - wenn es um Details geht - weitergeführt werden (vgl. Mai 2016, S. 125-128). Auf den Windschatten-Mechanismus zielt die Aussage, dass ,kein Minister eine wirksame Kontrolle über den unterstellten Apparat ausüben“ (Ellwein und Hesse 1987, S. 314) könne. Wenn es dennoch kaum zu „bureaucratic drift“ kommt, dann hat dies mit der als „Dialogmodell“ bezeichneten internen Kommunikationsstruktur zu tun, ,bei dem die Entscheidung und Weisungen der Spitze sich auf die Problemwahrnehmungen und Lösungsvorschläge der Basis stützen, ihrerseits aber wiederum die Aufmerksamkeit der Basis strukturieren und die Suche nach Lösungen leiten“" (Mayntz 1978, S. 193). Leitung und Arbeitsebene sind wechselseitig aufeinander angewiesen: Die Leitung benötigt die Expertise der Fachabteilungen, die ihrerseits ohne Zustimmung und Unterstützung der Leitung wenig durchsetzen können (vgl. Pfeiffer und Faller 1997, S. 35; Hustedt 2013, passim).

Die Erwartung, dass Minister/innen ihr Ressort gar nicht systematisch überwachen, sondern sich mit der Kontrolle von Resultaten begnügen, geht ursprünglich auf die Beobachtung einer ,relatively small institutional capacity at the top“ (Mayntz und Scharpf 1975, S. 86) zurück, die heute nicht mehr zutrifft. Während der Personalumfang der Bundesministerien zwischen 1980 und 2009 um rund $20 \%$ anstieg, nämlich von durchschnittlich 897 auf 1113 Mitarbeiter/innen, wuchsen die Leitungsstäbe im gleichen Zeitraum um 42\%, und zwar von durchschnittlich 38 auf 65 Personen (Zahlen gerundet nach Hustedt 2013, S. 241-245). Der Begriff „Leitungsstab“ war bis in die 1970er Jahre innerhalb der Bundesministerien ungebräuchlich. Die wenigen der Leitungsebene zugeordneten Referate spielten keine hervorgehobene Rolle. Das hat sich grundlegend geändert. Der nicht nur quantitative, sondern auch qualitati- 
ve Aufstieg der Leitungsstäbe, die heute mitunter eine ganze Abteilung bilden, ist einerseits auf den Bedeutungszuwachs der Presse- und Öffentlichkeitsarbeit zurückzuführen. Andererseits fungieren die Leitungsstäbe auch als Instanz zur Steuerung und Durchsetzung politischer Vorgaben innerhalb der Ressorts (vgl. dazu ausführlich: Hustedt 2013, S. 270-274, 327-335). Die Stärkung der Leitungsebene sowie die eng gewobenen Mitzeichnungs-, Kenntnisnahme- und Beteiligungsregeln ( $\S 15$, 19, 21, 24 GGO) sorgen dafür, dass einzelne Referate - etwa auf Veranlassung einer Interessengruppe - schwerlich im Alleingang politisch bedeutsame Vorhaben vorantreiben können. Die weiter unten präsentierten Befragungsergebnisse bestätigen diese Befunde.

Bei aller Unübersichtlichkeit der Konsultationspraxis lässt sich an den Organigrammen und Geschäftsverteilungsplänen ablesen, dass die Bundesministerien keineswegs ad hoc in Umweltkontakte ,hineinstolpern“. Entweder sind ganze Referate mit der dauerhaften Beobachtung der für das Ressort relevanten Verbändelandschaft betraut oder es sind spezielle Aufgaben im Kontakt mit Verbänden ausgewiesen. In die Zuständigkeit des Referats L11 (,,Koordinierung der Beteiligung von Verbänden“) des Verkehrsministeriums fällt bspw. die „,kontinuierliche[n] Kontaktpflege“ zu Fachverbänden, „Monitoring und Analyse der politischen, fachlichen und medialen Aktivitäten der Verbände im Geschäftsbereich“ sowie die „Analyse und Bewertung von Verbandspositionen und -aktivitäten zu politisch bedeutsamen Vorhaben des BMVI“". ${ }^{6}$ Im Geschäftsverteilungsplan des BMG sind nicht weniger als 19 Referate mit verbandsbezogenen Aufgaben aufgeführt. All dies deutet auf eine Professionalisierung hin, die sich nicht nur in ministeriell initiierten bzw. organisierten Kontakten niederschlägt, sondern auch im Aufbau von Kapazitäten für den strategischen Umgang mit Interessengruppen.

\subsection{Externe als Ersatzgesetzgeber}

Spätestens seit der Linklaters-Affäre aus dem Jahr 2009, bei der die gleichnamige Großkanzlei im Auftrag des BMWi einen ganzen Gesetzentwurf verfasste, ist die externe Beratung der Bundesministerien Gegenstand einer kontroversen Berichterstattung sowie juristischer Bedenken über ,die Gefahr der Umgehung und Entwertung der vom Grundgesetz vorgesehenen Verfahrenswege“ (Dreier 2018, S. 61). Auch der Bundesrechnungshof hat sich in bislang 14 Berichten und Prüfmitteilungen - meist kritisch - zu dieser Thematik geäußert. Insbesondere die von NGOs wie Lobby-Control oder Transparency Deutschland, den jeweiligen Oppositionsparteien oder den Medien erhobenen Vorwürfe, dass sich hinter der Nutzung externer Expertise ein verdeckter Klientelismus verberge, wirft die Frage auf, ob die verschiedenen (wissenschaftlichen) Beratungsformate, die Inanspruchnahme externer Politikformulierungshilfe sowie der Einsatz externer Personen in den Bundesministerien organisierten Interessen die Einflussnahme auf die Ministerialverwaltung erleichtern oder sich gar ein „Ersatzgesetzgeber“ (Schmieszek 2013) etablieren konnte.

Die älteste Quelle externer Beratung bilden die dauerhaft eingerichteten Beiräte und Sachverständigengremien, die von Geschäftsstellen der zuständigen Bundesmi-

\footnotetext{
${ }^{6}$ Die hier zitierten Geschäftsverteilungspläne finden sich auf der Homepage von https://fragdenstaat.de/.
} 
nisterien betreut werden, denen auch die Verarbeitung der dort entstehenden wissenschaftlichen Expertisen obliegt. Der letztmalig 2010 erschienene Gremienbericht der Bundesregierung führt insgesamt 143 Beiräte und Sachverständigengremien auf (vgl. Bundestag 2010, S. 24). In der Mehrzahl handelt es sich um gemischte Beratungsorgane, die nicht allein wissenschaftlich, sondern auch mit „Sachverständigen“ aus der Praxis besetzt sind, die den Interessen von Berufsgruppen oder Branchen nahestehen, z. T. auch aus Verbänden entsandt werden (vgl. Mayntz 1978, S. 205; Weber 1981, S. 283). Diese Form der Politikberatung ist kaum umstritten, was neben ihrem offiziellen Charakter und der routinemäßigen Veröffentlichung ihrer Arbeitsergebnisse auch Resultat der trägen Beratungsform des schriftlichen Gutachtens ist, welches zu einer Entkopplung vom politischen Tagesgeschehen beiträgt (vgl. Weingart und Lentsch 2008, S. 63; Döhler 2012). Bis zum Jahr 2000 war die Errichtung von Beiräten auf „Arbeitsgebiete von größerer Bedeutung“ ( $\$ 62$ Abs. 1 GGO I [a.F.]) beschränkt. Der Wegfall dieser Vorschrift deutet daraufhin, dass sich die Ressorts größere Freiräume bei der Errichtung von Beratungsgremien schaffen wollten (vgl. dazu Fleischer 2015). Dies ist mit der These kompatibel, dass es ,eher ein Instrumentalisierungsproblem für wissenschaftliche Berater als ein Autonomieproblem für die Beratenen“ (Mayntz 1978, S. 208) gäbe. Dass Ministerien wissenschaftliche Expertise nicht nur um der Erkenntnis willen, sondern vor allem zur Absicherung der eigenen Position nutzen, ist ein dazu passender empirischer Befund (vgl. Beneke 2019, S. 35-37). Teilweise ist die Ministerialverwaltung bereits im Entstehungsprozess der wissenschaftlichen Politikberatung präsent und nicht nur Adressat selbiger. So nimmt etwa das BMWi an den Beratungen der Wirtschaftsforschungsinstitute zur Erstellung der Gemeinschaftsprognose als „stiller Beobachter“ (Campbell und Pedersen 2014, S. 152) teil, ist dadurch vor Überraschungen geschützt und trägt mit einer eigenen, jährlich stattfindenden Konferenz zur Meinungsbildung in der Konjunkturforschung bei (vgl. Campbell und Pedersen 2014, S. 153-154).

Im Gegensatz zur institutionalisierten Beratung ist die meist ad hoc stattfindende Nutzung privater Dienstleister seit Mitte der 2000er als „Berater-Republik“ (Zeit 2004) Gegenstand anhaltender Kritik. ${ }^{7}$ Die rot-grüne Koalition hat die Inanspruchnahme von Unternehmensberatungen, Anwaltskanzleien oder Public Affairs-Agenturen anfänglich offensiv als Modernitätssignal zu verteidigen versucht, wonach die überlegene Effizienz und Geschwindigkeit des privaten Sektors für politische Vorhaben eingesetzt und damit gesamtgesellschaftlicher Nutzen erzielbar sei (vgl. z.B. Bundestag 2004). Diese Deutung konnte sich gegen die - häufig in pompösem Entlarvungsgestus formulierte - Kritik von Oppositionsparteien, ,neuartigen Moralunternehme[n]“ (Wewer 2015, S. 43), inklusive dem Bund der Steuerzah-

\footnotetext{
7 Vgl. z. B. FAZ (2013). Die in der Wirtschaftswoche (2019) genannten „,mehr als 1,2 Mrd. EUR“, die seit 2006 für „Regierungsberater“ aufgewandt worden seien, beinhalten Fördermittel des Forschungsministeriums in Höhe von 465 Mio. EUR, die von den Projektträgern verwaltet werden. Selbst wenn in Vorhaben der Bundesregierung auf Forschungsergebnisse Bezug genommen würde, die aus Mitteln des BMBF stammen, ist die Überschrift „Regierungsberater“ für diese Ausgaben grob irreführend. Zudem offenbart eine einfache Rechenoperation den ganzen Unsinn der in diesem Kontext gern benutzten Aggregatzahlen. Dividiert man die von 1,2 Mrd. EUR verbliebenen 735 Mio. EUR durch den Beobachtungszeitraum von dreizehn Jahren und die Summe von 56,5 Mio. EUR wiederum durch die Anzahl der Ressorts, nämlich 14, ergibt dies den wenig schlagzeilenträchtigen Betrag von rund 4Mio. EUR pro Jahr und Ministerium.
} 
ler, und einem sich daraus speisenden medialen Überbietungswettbewerb, wer über die größte Steuerverschwendung oder das mächtigste Trojanische Pferd im Staatsapparat berichten konnte, nicht durchsetzen. Dazu haben Bundesregierungen aller Couleur aber auch mit zögerlicher Informationsweitergabe, beanstandungswürdigen Vergabeverfahren und wenig überzeugenden Begründungen beigetragen (vgl. etwa Süddeutsche 2016).

In der jüngeren Vergangenheit stand die als Gesetzgebungsoutsourcing in Verruf geratene „Mandatierung gegen Entgelt im Vorbereitungsstadium“ (Schmieszek 2013, S. 175) sowie der als ,institutionalisierter Lobbyismus“ (Bundestag 2007) bezeichnete Einsatz externer Personen innerhalb der Bundesministerien im Zentrum der Aufmerksamkeit. Dass dieses verfassungsrechtlich zwar nicht zu beanstandende (vgl. Ossenbühl 2012), aber dennoch unübliche Procedere als ,Beschädigung des Prinzips einer sachorientierten, unparteiischen Entscheidungsvorbereitung“ (Müller 2013, S. 64) bewertet wird, mag verständlich sein, ignoriert aber auch die Ursachen der Externalisierung. Als unstrittig kann gelten, dass sich die Bundesministerien zunehmend mit Problemen konfrontiert sehen, deren Komplexität und Änderungsgeschwindigkeit allein mit der eigenen Fach- und vor allem Detail-Expertise nicht mehr lösbar sind (vgl. Goetz 2007, S. 177-182; Döhler 2012; Loer und Töller 2019). Die wenig sensible, aber plausible Äußerung des damaligen Finanzministers Steinbrück, sein Ressort könne ,nicht noch einen Experten für Kirschblütenbestäubung vorhalten“ (Spiegel 2009), beschreibt das Dilemma, dass die begrenzten Ressourcen eines Bundesministeriums nicht immer mit dem Komplexitätszuwachs der Regelungsgegenstände in Einklang gebracht werden können. Die Inanspruchnahme Externer ist daher als Versuch zu verstehen, Expertise-Defizite auszugleichen oder Zeitdruck zu überwinden (vgl. Döhler 2012, S. 197-200; Schmieszek 2013, S. 178; Kalagi 2014, S. 654-659). Auch wenn Kritiker kaum davon zu überzeugen seien werden, dass externe Beratungsleistungen nicht ,ehrfurchtsvoll und staunend ab[ge]nick[t]“ (Schmieszek 2013, S. 176) werden, spricht wenig dafür, dass die Ministerialverwaltung etwas anderes als die fachliche Unterstützung eigener Ideen einkauft.

Über die Gesetzgebung auf Honorarbasis liegen seit 2009 keine neuen Zahlen vor (näheres dazu bei Döhler 2012, S. 194-197). Vermutlich agieren die Bundesministerien bei der Nutzung von Anwaltskanzleien vorsichtiger und greifen bei der Inanspruchnahme externer Beratung auf den unspezifischen „Abruf aus Rahmenvertrag“ zurück - so eine gängige Antwort auf Bundestagsanfragen. Medial beinahe bedauernd zur Kenntnis genommen wird der Umstand, dass die Zahl externer Personen im Sinken begriffen ist (vgl. TAZ 2013). Die Mutmaßung, dass auf diesem Wege massiv Einfluss genommen wird, war schon deshalb nie besonders überzeugend, weil die Anzahl (zuletzt 32 externe Personen, verteilt auf 14 Ministerien mit rund 20.000 Beschäftigten) und auch deren Herkunft (zuletzt eine Person aus der Privatwirtschaft) ministerielle Arbeitsprozesse und deren Inhalte schwerlich in spürbarem Umfang beeinflussen können. Das Thema hat dadurch weiter an Brisanz verloren, dass die bis 2014 als Ausschussdrucksachen der Öffentlichkeit nicht zugängigen „Berichte über den Einsatz externer Personen in der Bundesverwaltung“ mittlerweile auf der Homepage des BMI veröffentlicht werden. Auch wenn vereinzelt Formulierungen von Interessengruppen ihren Weg in gesetzliche Reglungen finden (vgl. u. a. Handelsblatt 2013; ZEIT 2013; Naggert 2018, S. 354-399), steht 
die verbreitete Interpretation auf schwachen Füßen, dass darin ein generalisierbarer Beleg für den Erfolg externer Interessen gesehen werden könne. Abgesehen von der geringen Fallzahl spricht die hier präsentierte Analyse eher für die Annahme, dass die Ministerialverwaltung nur für solche Vorschläge empfänglich ist, die zu ihren Lösungskonzepten passen.

\section{Einstellungen der Ministerialverwaltung zum Umgang mit Interessengruppen}

Wenn, wie weiter oben ausgeführt, die Informationsabhängigkeit der Ministerialverwaltung überschätzt wird und sich offenkundiger Klientelismus in Grenzen hält, bleibt die Frage, aus welchen Motiven sich Ministerien jenseits der Indienstnahme korporativer Akteure dann überhaupt für Verbände öffnen sollten. Aus der jüngeren Forschung über Interessenvermittlung auf der EU-Ebene kann bei dieser Frage auf das Konzept der ,,access goods“ zurückgegriffen werden. Demnach hängt der Erfolg von Verbänden davon ab, welche Zugangsgüter sie politischen Entscheidungsträgern anbieten können. Dabei lassen sich drei Typen von Zugangsgütern unterscheiden (vgl. Bouwen 2004, S. 340): Erstens das Vollzugswissen, das branchenspezifische Geschäftsabläufe oder technische Funktionsdetails umfasst und den wesentlichen Anlass für die Nutzung externer Politikformulierungshilfe bildet (vgl. Döhler 2012), zweitens die Antizipation von Widerständen und drittens die Unterstützung politischer Vorhaben. Würde man erwarten, dass diese in der Interaktion mit Verbänden erhältlichen Informationen von Ministerien ignoriert oder für unwichtig befunden werden, müsste man ein Maß an Naivität oder Leichtfertigkeit unterstellen, für das empirisch keine Belege vorhanden sind. Das Konzept der Zugangsgüter, das zweifellos um weitere Kategorien wie Vertrauen oder Legitimation erweiterbar ist, stellt aber auch deshalb eine wichtige Neuerung dar, weil das Verhältnis zwischen Staat und Verbänden nicht als einseitiger Vorgang konzipiert wird, sondern - wie bereits in der Korporatismusdebatte - als Tauschprozess, nunmehr allerdings mit veränderlichen „Geschäftsbedingungen“, die dazu führen können, dass Verbände ignoriert werden bzw. gegen deren Interessen gehandelt wird (für einige Beispiele vgl. Handelsblatt 2010).

Dass sich die Wahrnehmung der Ministerialverwaltung gegenüber Interessengruppen tatsächlich ändert, kann anhand des Surveys „Politisch-Administrative Elite“ $(\mathrm{PAE})^{8}$ aufgezeigt werden. Auf eine Differenzierung nach Ministerien, die an einigen

\footnotetext{
8 Die Online-Befragung PAE 2017 ist die Fortführung einer 1972 erstmals von einer amerikanischen Forschergruppe und seit 2005 jeweils kurz vor der Bundestagswahl durchgeführten Befragung über die sozialstrukturellen Merkmale (Herkunft, Ausbildung und Hochschulbildung, Karrierewege), die Politisierung sowie das Politik- und Rollenverständnis leitender Mitarbeiter/innen aller Bundesministerien, ausgenommen das Verteidigungsministerium. Befragt wurden jeweils alle Unterabteilungs- und Abteilungsleiter/ innen sowie die beamteten Staatssekretäre/innen, ab 2013 auch die Referatsleiter/innen. 2017 waren dies insgesamt 1905 Personen. Die Rücklaufquote im Jahr 2017 betrug 34,0\% (N=649), wobei 210 Fragebögen nicht vollständig beantwortet wurden. Näheres findet sich auf der Homepage des Autors, der seit 2017 zusammen mit Falk Ebinger und Sylvia Veit zum PAE-Team gehört. Nachfolgende Zitate ohne Literaturangabe sind dem PAE-Fragebogen entnommen.
} 
Stellen eine tiefenschärfere Analyse erlauben würde (vgl. etwa Beneke 2019), wird hier verzichtet, um generelle, auf die Gesamtheit der Bundesministerien anwendbare Aussagen zu gewinnen. Das Rollenverständnis der Ministerialverwaltung erlaubt keine direkten, wohl aber indirekte Schlüsse über den Umgang mit Interessengruppen und dessen Wandel. Auf die Frage, welchem Rollentypus sich die Mitarbeiter/ innen zugehörig fühlen, erhält auf einer vierstufigen Skala der „Experte mit Fachwissen für Problemlösungen“ die höchste Anzahl von Nennungen, nämlich 61,3\% (,trifft ganz zu“) bzw. 31,1\% (,trifft etwas zu“). An zweiter Stelle folgt der Typus „Umsetzer politischer Vorgaben“ mit 37,7\% bzw. 48,4\%. Die meisten Nennungen in der Kategorie „trifft ganz zu“ erhält mit 45,3\% der „Repräsentant des Staates“. Eine ebenfalls hohe Zahl der Befragten, nämlich 26,4\% bzw. 51,9\%, sieht sich als „,Vermittler zwischen konfligierenden Interessen“. Demgegenüber verstehen sich nur $9,0 \%$ bzw. 35,4\% als „Fürsprecher breiter gesellschaftlicher Gruppen“. Auch wenn die letztgenannte Antwortkategorie einiges offenlässt, ist erkennbar, dass die Ministerialverwaltung sich nicht als Vertreter singulärer Interessen versteht, sondern ein deutlich breiteres Selbstverständnis besitzt. Gefragt nach denjenigen Tätigkeiten, die den größten Teil der Arbeitszeit beansprucht, geben 63,8\% die „Bearbeitung von E-Mails“ an. Daraus geht nicht hervor, welchen Umfang die Kommunikation mit organisierten Interessen besitzt. Eine Tendenz lässt jedoch die Antwort auf die Frage erkennen, wie häufig Kontakte zu Interessengruppen bestehen (vgl. Abb. 1). Nur 6,2\% bzw. 10,1\% der Befragten stehen mehrmals bzw. einmal pro Woche mit

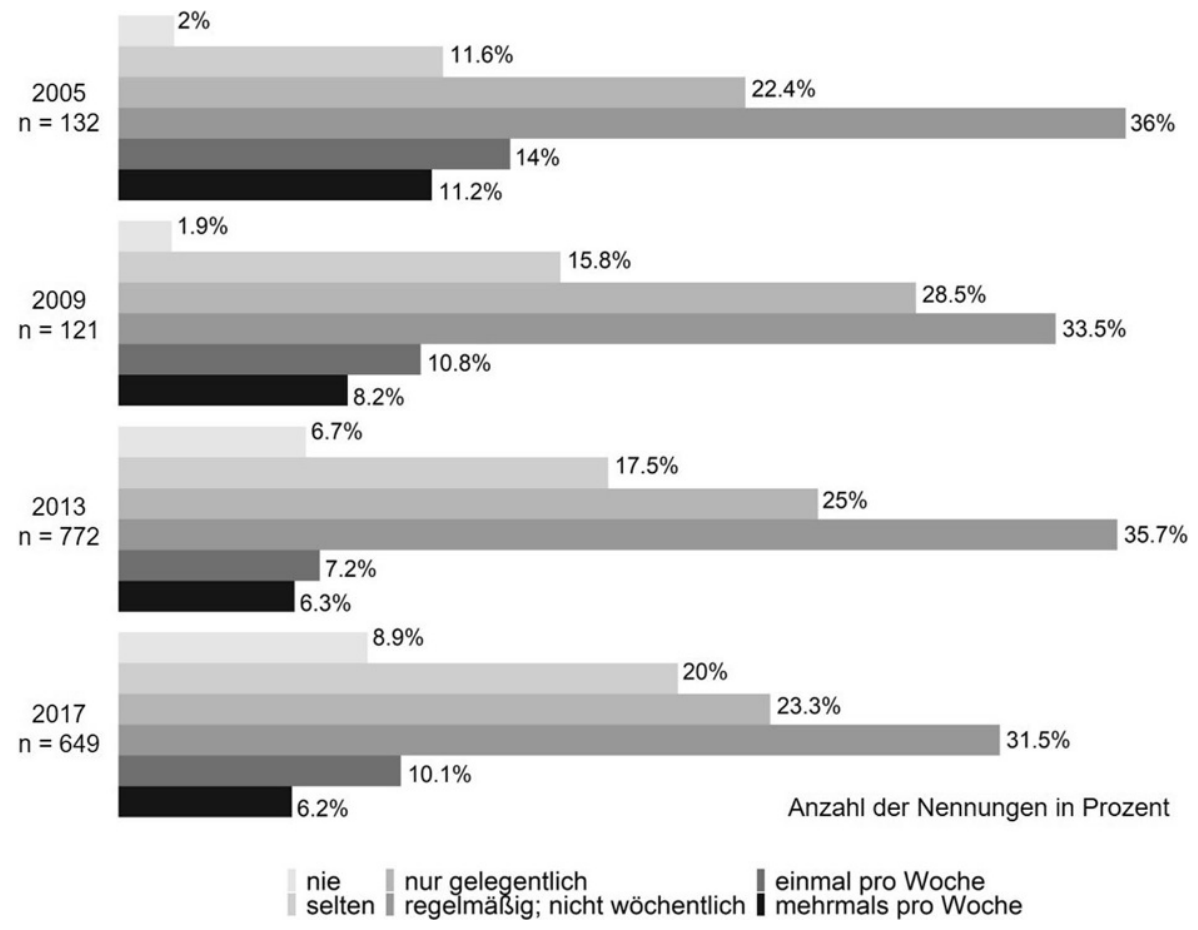

Abb. 1 Kontakthäufigkeiten zu Verbänden 


\section{Extern}

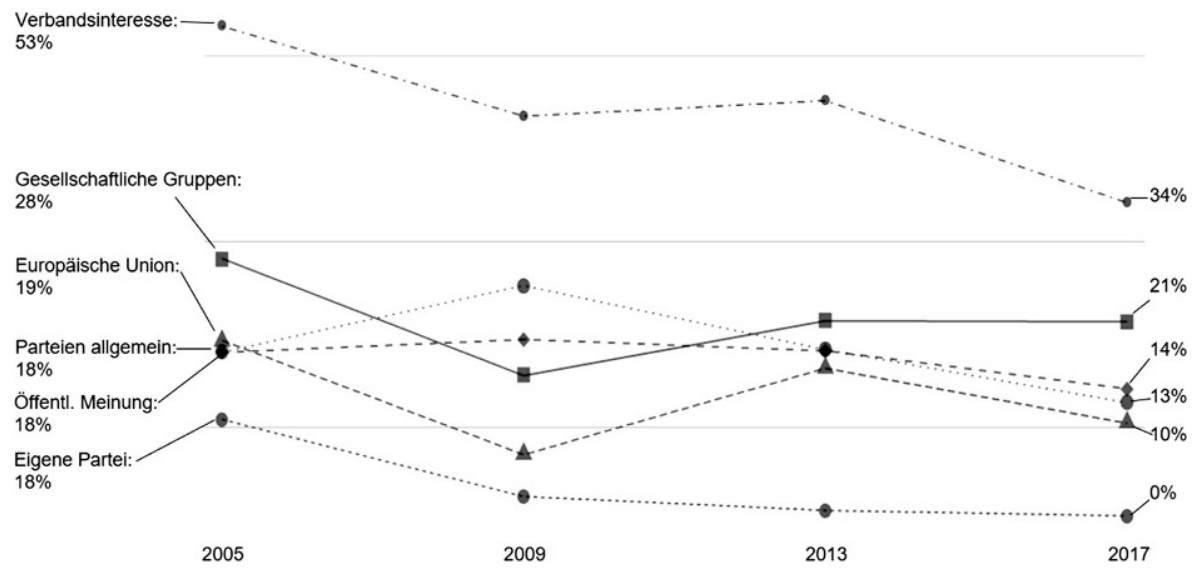

Intern

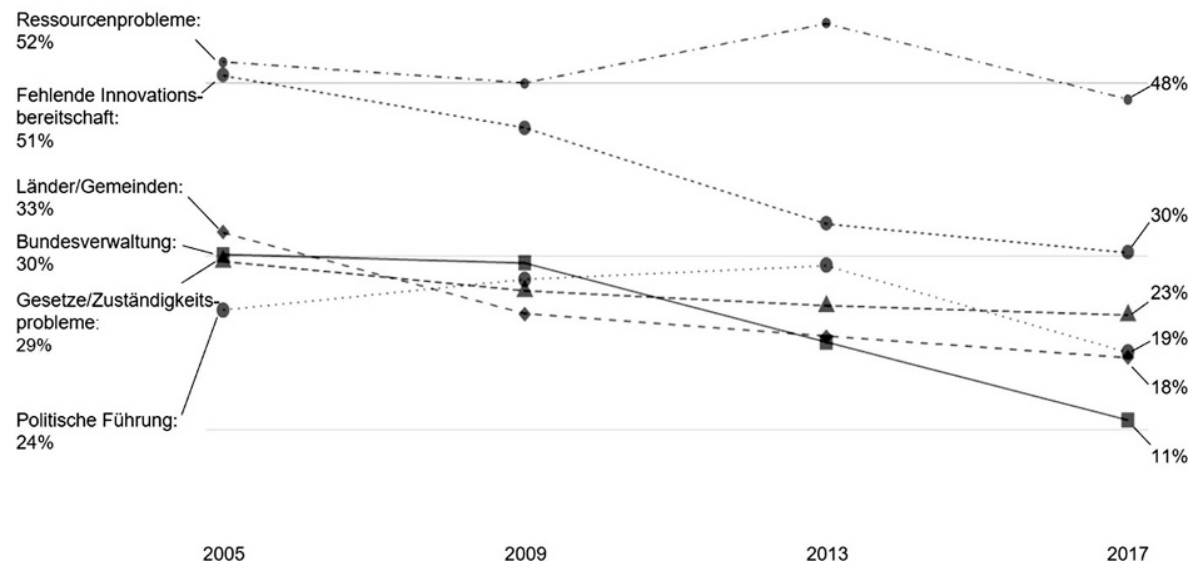

Abb. 2 Widerstände bei Problemlösungen

Verbänden in Kontakt. Aus den Angaben über die Kontakthäufigkeiten lässt sich folgern, dass Verbände im Arbeitsalltag der Ministerialverwaltung eine eher mittelgroße bzw. seit 2005 sogar abnehmende Rolle spielen.

Dieser Schluss drängt sich auch bei den Antworten auf folgende Frage auf (vgl. Abb. 2): „Wenn Sie an das wichtigste in Ihrem Tätigkeitsbereich liegende Problem denken, auf welche Widerstände stößt man beim Versuch, dieses Problem anzugehen?" Während Ressourcenprobleme stabil an erster Stelle rangieren, lässt sich das deutliche Absinken bei der Nennung von Verbänden von 53,2\% im Jahr 2005 auf 34,2\% im Jahr 2017 als Bestätigung der Zerfaserungsthese der Verbändeforschung werten, der zufolge die Ausdifferenzierung organisierter Interessen zwar mit zahlenmäßigem Wachstum, nicht aber mit erhöhter Durchsetzungsfähigkeit einhergeht.

Auf die Frage, welche Akteure den größten Einfluss auf bundespolitische Entscheidungen besitzen, geben die meisten Befragten auf einer Skala von eins (,,sehr 
groß“) bis fünf („,nicht relevant“) die jeweilige Ministeriumsleitung mit 60,2\% (sehr groß) bzw. 34,6\% (groß) an. Dies bestätigt, dass die Ministerialverwaltung kaum zu Eigenmächtigkeiten neigt, sondern die „kritische Loyalität“ (Veit et al. 2018, S. 410) das Selbstverständnis bestimmt. Die zweithäufigste Nennung gilt „führenden Mitgliedern der Parteien“, denen 61,1\% der Befragten einen sehr großen und $29,6 \%$ einen großen Einfluss zurechnen. 2017 sahen (ebenso wie 2005) nur 10,2\% einen sehr großen, aber noch 44,5\% einen großen Einfluss bei Verbänden. Inwieweit letztere Angabe dadurch relativiert werden müsste, dass Arbeitgeberverbände und Gewerkschaften als gesonderte Antwortmöglichkeit aufgeführt sind, ist schwer zu beurteilen. 14,2\% rechnen den Arbeitsgebern einen sehr großen Einfluss zu, groBen Einfluss noch 43,0\%. Gewerkschaften schneiden mit 9,3\% bzw. 37,9\% etwas schwächer ab. Dass der „Presse“ mit 26,4\% bzw. 41,8\% sowie den „Sozialen Medien“ mit 6,1\% bzw. 26,1\% ein sehr großer bzw. ein großer Einfluss zugerechnet wird, zeigt, dass Interessengruppen nicht an erster Stelle stehen, wenn es um die Bewertung politischer Einflusspotentiale geht. So lassen sich auch die Antworten auf die Frage interpretieren, wer zukünftig mehr oder weniger politischen Einfluss haben sollte. 64,2\% wünschen sich weniger Einfluss der „Privatwirtschaft/Banken“, 55,2\% weniger Einfluss der sozialen Medien und 46,6\% weniger Einfluss der Presse. Verbände rangieren in dieser Kategorie bei immerhin $51,8 \%$, was auf deren mutmaßliches Störpotential schließen lässt.

Die Befragungsergebnisse machen deutlich, dass die fachliche Expertise, die Folgebereitschaft gegenüber der Leitungsebene (so auch Veit et al. 2018) sowie die Vermittlerrolle im Angesicht konkurrierender Verbandsinteressen das Selbstverständnis der bundesdeutschen Ministerialverwaltung am stärksten prägen. Das schließt externe Einflüsse nicht aus. Aber Forderungen und Wünsche, die von Verbänden oder zivilgesellschaftlichen Akteuren erhoben werden, müssen immer erst die Filter des ministeriellen Rollenverständnisses durchlaufen. Interessengruppen stehen überdies auch nicht im Zentrum der Aufmerksamkeit. Sie werden zwar als relevant, teilweise auch als Störfaktor wahrgenommen, rangieren bei den Außenkontakten sowie im Alltagsgeschäft aber hinter der Spitze des eigenen Hauses, der Presse und den sozialen Medien. Die Interaktionen zwischen Ministerialverwaltung und Verbänden lassen sich nun unter Zugrundelegung der drei Analysedimensionen folgendermaßen abbilden (Abb. 3).

Die Makroebene bildet den Ort des Kontakts zwischen Ministerien und Interessengruppen, wo sowohl Einflussversuche ihren Ausgang nehmen wie auch umgekehrt die Instrumentalisierung von Interessengruppen für politische Ziele stattfindet. Dass externe Einflüsse auf die Bundesministerien in diesem Prozess nicht ungefiltert bleiben, ist ein wesentliches, aber zu selten beachtetes Ergebnis der vorliegenden Analyse. Während die Zugangsgüter wie Eintrittskarten zur ministeriellen Interessenberücksichtigung wirken, durchlaufen Verbändeforderungen in der Organisationsdimension verschiedene formale und prozedurale Filter, bevor sie mit dem dreistufigen Rollenverständnis der Ministerialverwaltung konfrontiert und dabei weiter - mehr oder minder stark - modifiziert werden. Dieses Schema ist stark vereinfacht, erlaubt aber eine analytisch präzisere Erfassung von Interaktionen, als wenn diese lediglich summarisch als „Einflussnahme“ oder „Lobbying“ charakterisiert werden. 


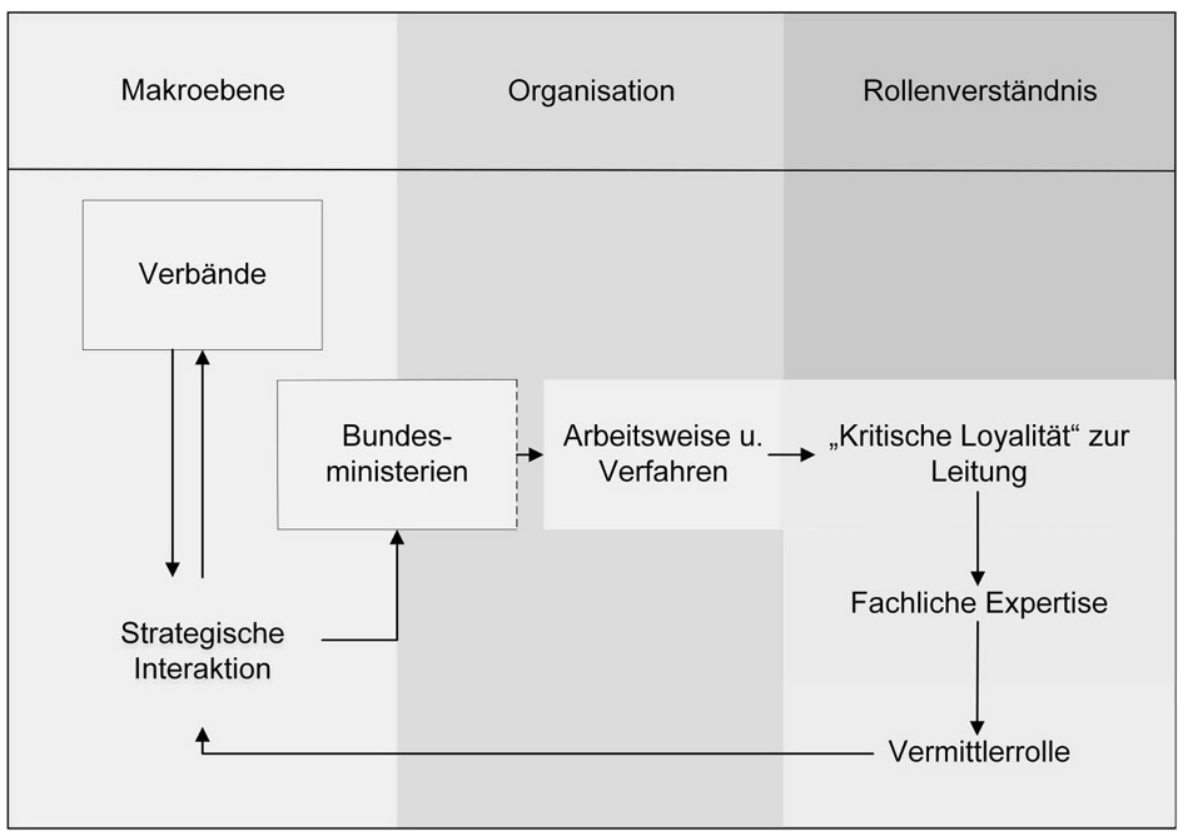

Abb. 3 Interaktionsschema zwischen Ministerialverwaltung und Verbänden

\section{Zusammenfassung}

Der Argwohn, der die Diskussion über die Teilnahme von Verbänden am politischen Prozess hierzulande begleitet, ist Ausfluss eines nicht mehr explizit vertretenen, aber immer noch wirkmächtigen Idealmodells, das eine Trennung zwischen Staat und Gesellschaft vorsieht. Außenkontakte der Verwaltung, die das Gemeinwohl vertreten soll, geraten daher leicht in den Verdacht, Sonderinteressen zu bevorzugen. Diese speziell mit dem Lobbyismus-Konzept verbundene Wirkungsvermutung setzt aber nicht nur voraus, dass Einzelinteressen konkurrenzarm artikuliert werden, sondern auch, dass die Adressaten, unter ihnen die Ministerialverwaltung, kaum über eine Notariats-Funktion hinauskommen. Im Gegensatz dazu spricht die hier vorgelegte Analyse dafür, dass die Ministerialverwaltung externe Einflussbestrebungen nicht erdulden muss, sondern eigene strategische Kompetenz für den Umgang mit organisierten Interessen entwickelt hat. Damit kann ein realitätsnäheres Bild gezeichnet werden als im dominierenden Lobbyismus-Diskurs. Die Politikwissenschaft ist an dessen Dominanz nicht ganz unschuldig, da zu wenig alternative Konzepte entwickelt werden, die einen Erkenntnisgewinn versprechen. Effekte wie Staatsentlastung, Konsensbildung oder die Steigerung politisch-administrativer Handlungsfähigkeit bilden sicher nicht die komplette Bandbreite der Staat-Verbände-Beziehungen ab. Aber eine Rückbesinnung - wohlgemerkt: keine Wiederbelebung - auf den Erkenntnisstand der Korporatismus-Diskussion könnte nicht nur die innovationsarme Verbändeforschung beleben, sondern auch einen Beitrag zur Wiedererlangung der 
Deutungshoheit in einem von Steuerungs- und Politikpessimismus geprägten Feld leisten.

- In der ersten der drei Analysedimensionen, die die Makroebene des Verhältnisses zwischen Verbänden und Ministerialverwaltung beschreibt, wird explizit auf Überlegungen aus der Korporatismus-Diskussion Bezug genommen. Mit der Betonung einer Einflussumkehr, den Konzepten der Interessenvermittlung und der Verpflichtungsfähigkeit von Verbänden ist seinerzeit eine Blaupause entstanden, die auch heute die Analyse der Staat-Verbände-Beziehungen befruchten kann. Dies gilt insbesondere für die Erklärung unterschiedlicher Kontakt- bzw. Kooperationsformen zwischen Ministerialverwaltung und Interessengruppen, die von der finanziellen Unterstützung, über die missions- bzw. strukturgetriebene Einflussnahme auf die Organisation von Verbänden, bis hin zu den Stakeholder-Dialogen reichen. Angesichts dieser Variabilität liegt die Vermutung nahe, dass sich das gewählte Format strategisch an strukturelle Gegebenheiten der Verbändelandschaft bzw. deren Wandel anpasst.

- Die zweite Analysedimension gilt jenen Faktoren, die das Verhältnis zu Verbänden auf der organisatorischen Ebene prägen. Weder sind die formalen Regeln für den Umgang mit Interessengruppen noch Organisation und Arbeitsweise der Bundesministerien so beschaffen, dass sie bloße Einfallstore für externe Interessen bilden. Die oft geltend gemachte Informationsabhängigkeit ist zudem weniger ausgeprägt als behauptet und kann ebenso wie die Inanspruchnahme externer Politikformulierungshilfe weder vom Umfang noch von ihrer Veranlassung her als Beleg für lobbyistisches Durchregieren betrachtet werden.

- Die dritte Analysedimension schließlich widmet sich dem Personal der Bundesministerien und dessen Einstellungen. Das Konstrukt der Zugangsgüter besagt, dass Verbände einen akteurspezifischen Tauschwert anbieten müssen, um sich erfolgversprechend artikulieren zu können. Für die Bundesministerien stehen dabei insbesondere das Vollzugs- und Antizipationswissen im Vordergrund. Die Ergebnisse der PAE-Studie erhellen komplementär dazu die Motivlage der Ministerialverwaltung im Umgang mit Verbänden. Hervorhebenswert ist das ministerielle Rollenverständnis, das einer undifferenzierten Interessenberücksichtigung entgegenwirkt. Verbände und ihre Aktivitäten werden sehr wohl wahrgenommen, bilden aber nicht die zentrale Orientierungsmarke, sondern stehen im Prozess der Politikformulierung in Konkurrenz zu anderen, insbesondere hierarchischen und medialen Einflussfaktoren.

Insgesamt führt diese dreidimensionale Analyse zu dem Ergebnis, dass im Verhältnis zwischen Ministerialverwaltung und Interessengruppen grundsätzlich von einer reziproken Beziehung auszugehen ist. Die Ministerialverwaltung kann auf die Zugangsoptionen, die Struktur und die finanzielle Ausstattung organisierter Interessen einwirken. Umgekehrt sind externe Einflüsse vielfältig, müssen ihrerseits aber immer die Filter der formalen Regeln, der Binnenorganisation und Arbeitsweise sowie des Rollenverständnisses der Ministerialverwaltung durchlaufen, bevor sie eine Wirkung erzielen können. Dass sich Verbände in der Politikformulierung mitunter 
auch gegen ministerielle Präferenzen durchsetzen, wird damit nicht bestritten, wohl aber, dass dies voraussetzungsarm oder gar automatisch geschieht.

Funding Open Access funding provided by Projekt DEAL.

Open Access Dieser Artikel wird unter der Creative Commons Namensnennung 4.0 International Lizenz veröffentlicht, welche die Nutzung, Vervielfältigung, Bearbeitung, Verbreitung und Wiedergabe in jeglichem Medium und Format erlaubt, sofern Sie den/die ursprünglichen Autor(en) und die Quelle ordnungsgemäß nennen, einen Link zur Creative Commons Lizenz beifügen und angeben, ob Änderungen vorgenommen wurden.

Die in diesem Artikel enthaltenen Bilder und sonstiges Drittmaterial unterliegen ebenfalls der genannten Creative Commons Lizenz, sofern sich aus der Abbildungslegende nichts anderes ergibt. Sofern das betreffende Material nicht unter der genannten Creative Commons Lizenz steht und die betreffende Handlung nicht nach gesetzlichen Vorschriften erlaubt ist, ist für die oben aufgeführten Weiterverwendungen des Materials die Einwilligung des jeweiligen Rechteinhabers einzuholen.

Weitere Details zur Lizenz entnehmen Sie bitte der Lizenzinformation auf http://creativecommons.org/ licenses/by/4.0/deed.de.

\section{Literatur}

Adam, Matthias. 2004. Neue Wege in der Forschungsförderung? Forschungsjournal Soziale Bewegungen 17(2):85-90.

Bach, Tobias. 2012. The involvement of agencies in policy formulation: explaining variation in policy autonomy of federal agencies in Germany. Policy and Society 31(3):211-222.

Backhaus-Maul, Holger, und Thomas Olk. 1994. Von Subsidiarität zu „outcontracting“: Zum Wandel der Beziehungen von Staat und Wohlfahrtsverbänden in der Sozialpolitik. In Staat und Verbände Politische Vierteljahresschrift Sonderheft, Bd. 25, Hrsg. Wolfgang Streeck, 100-135. Opladen: Westdeutscher Verlag.

Baruth, Stephanie, und Kai-Uwe Schnapp. 2015. Ministerialbürokratien als Lobbyadressaten. In Lobby Work: Interessenvertretung als Politikgestaltung, Hrsg. Rudolf Speth, Annette Zimmer, 245-260. Wiesbaden: Springer VS.

BDI. 2008. Landwirtschaftliche Sozialversicherung (LSV): Bundesregierung scheint das Wort „Spitzenverband" zu lieben. : Berufsverband Deutscher Internisten. http://bdi.bsmo.de/bdi/content/020/010/ 0710111.jsp

Beneke, Jasmin Theres. 2019. Wissenschaftliche Beratung der Bundesministerien - ein Politikfeldvergleich. unveröff. MA-Arbeit, Leibniz Universität Hannover.

Blätte, Andreas. 2010. Islamische Verbände in verbandsökonomischer Perspektive., 22-28. Heinrich-BöllStiftung: Muslimische Gemeinschaften zwischen Recht und Politik.

Bouwen, Pieter. 2004. Exchanging access goods for access: a comparative study of business lobbying in the European Union institutions. European Journal of Political Research 43(3):337-369.

Braun, Sebastian, und Doreen Reymann. 2013. Der DOSB als Dachorganisation des vereins-und verbandsorganisierten Sports in Deutschland. In Der Deutsche Olympische Sportbund in der Zivilgesellschaft, Hrsg. Sebastian Braun, 33-39. Wiesbaden: Springer VS.

Bundesrechnungshof. 2014. Abschließende Mitteilung an das Bundesministerium des Inneren über die Prüfung Förderung der Spitzenverbände. Bonn: Bundesrechnungshof.

Campbell, John L., und Ove K. Pedersen. 2014. The national origins of policy ideas: Knowledge regimes in the United States, France, Germany, and Denmark. Princeton: Princeton University Press.

Czada, Roland. 1991. Regierung und Verwaltung als Organisatoren gesellschaftlicher Interessen. In $R e$ gieren in der Bundesrepublik III, Hrsg. Hans-Hermann Hartwich, Göttrik Wewer, 151-173. Opladen: Leske + Budrich.

Czada, Roland. 1994. Konjunkturen des Korporatismus: Zur Geschichte eines Paradigmenwechsels in der Verbändeforschung. Politische Vierteljahresschrift Sonderheft 25: Staat und Verbände 25:37-64. 
Czada, Roland. 2015. Die deutsche Verhandlungsdemokratie: Diskursform oder Elitenkartell? In Die Verhandlungsdemokratie. Dialogische Entscheidungsverfahren in der Politik, Hrsg. Thomas Mayer, Udo Vorholt, 11-36. Bochum, Freiburg: projektverlag.

Der Spiegel. 2009. Die verdrängten Sünden der Heuschrecken-Bändiger

Deutscher Bundestag. 2004. Antwort der Bundesregierung auf die Kleine Anfrage [...] der Fraktion der CDU/CSU - Drucksache 15/2365 - Vergabepraxis und Kosten externer Beratung der Bundesregierung. Berlin: Deutscher Bundestag.

Deutscher Bundestag. 2007. Antwort der Bundesregierungauf die Kleine Anfrage der (...) Fraktion DIE LINKE. Drucksache 16/5203 - Institutionalisierter Lobbyismus. Berlin: Deutscher Bundestag.

Deutscher Bundestag. 2010. Fünfter Gremienbericht der Bundesregierung zum Bundesgremienbesetzungsgesetz (Berichtszeitraum: 30. Juni 2005 bis 30. Juni 2009). In Drucksache 17/4318.

Deutscher Bundestag. 2018. Bundestags-Drucksache 19/2376: Antwort der Bundesregierung auf die Kleine Anfrage [...] der Fraktion Bündnis 90/Die Grünen. Förderung der Selbstorganisation von Migrantinnen und Migranten. Berlin: Deutscher Bundestag.

Deutscher Bundestag. 2019a. Bundestags-Drucksache 19/9865. Antwort der Bundesregierung auf die Kleine Anfrage [...] der Fraktion der FDP: Zusammenarbeit von Bundesregierung und externen Interessenträgern (Teil 12) - WWF. Berlin: Deutscher Bundestag.

Deutscher Bundestag. 2019b. Drucksache 19/7494. Antwort der Bundesregierung auf die kleine Anfrage [...] der Fraktion der FDP - Das bundesdeutsche „Gipfelwesen“. Berlin: Deutscher Bundestag.

DIE ZEIT. 2004. Die Berater-Republik. 5. Febr. 2004.

DIE ZEIT. 2013. Ökolabel für Autos: Brüssel soll Einfluss der Autolobby klären. 28. Okt. 2013.

Döhler, Marian. 2007. Die politische Steuerung der Verwaltung. Eine empirische Studie über politischadministrative Interaktionen auf der Bundesebene. Baden-Baden: Nomos.

Döhler, Marian. 2012. Gesetzgebung auf Honorarbasis - Politik, Ministerialverwaltung und das Problem externer Beteiligung an Rechtsetzungsprozessen. Politische Vierteljahresschrift 53(2):181-210.

Döhler, Marian. 2017. The bias of structures: how multidivisional organizations work in the public sector. Public Organization Review 17(1):83-100.

Döhler, Marian, und Philip Manow. 1992. Korporatisierung als gesundheitspolitische Strategie. Staatswissenschaften und Staatspraxis 3(1):64-106.

Döhler, Marian, und Philip Manow. 1997. Strukturbildung von Politikfeldern: das Beispiel bundesdeutscher Gesundheitspolitik seit den fünfziger Jahren. Opladen: Leske + Budrich.

Dreier, Horst. 2018. Vom Schwinden der Demokratie. In Die Zukunft der Demokratie, Hrsg. Graf Friedrich Wilhelm, Heinrich Meier, 29-81. München: C.H. Beck.

Ellwein, Thomas, und Joachim-Jens Hesse. 1987. Das Regierungssystem der Bundesrepublik Deutschland, 6. Aufl., Wiesbaden: Westdeutscher Verlag.

Fleischer, Julia. 2015. Organisierte Expertise und die Legitimation der Verwaltung: Sektorale und strukturpolitische Dynamiken der Gremienlandschaft auf Bundesebene. der moderne staat 8(2):315-335.

Frankfurter Allgemeine Zeitung. 2013. In vier Jahren: Ministerien zahlten fast $1 \mathrm{Mrd} . €$ an Berater. 10. Sept. 2013.

Fraussen, Bert. 2014. The visible hand of the state: on the organizational development of interest groups. Public Administration 92(2):406-421.

Goetz, Klaus. 2007. German officials and the federal policy process: the decline of sectional leadership. In From the active to the enabling state, Hrsg. Edward C. Page, Vincent Wright, 164-188. Houndmills: Palgrave Macmillan.

Göhler, Gerhard, Katrin Grothe, Cornelia Schmalz-Jacobsen, und Christian Walther. 2012. Public Affairs. Die neue Welt des Lobbyismus. Frankfurt/M.: Peter Lang.

Handelsblatt. 2010. Wo sich die Lobbyisten schwer tun. 7. Sept. 2010.

Handelsblatt. 2013. Lebensversicherungen: Versicherungslobby schrieb Gesetzentwurf. 18. Febr. 2013.

Hebeler, Timo. 2018. Ausweitung der gewerkschaftlichen Beteiligung bei der Vorbereitung gesetzlicher Regelungen der beamtenrechtlichen Verhältnisse? Neue Zeitschrift für Verwaltungsrecht 37(18):1337-1416.

Heinze, Rolf G. 2002. Die Berliner Räterepublik.: Viel Rat-wenig Tat? Wiesbaden: Westdeutscher Verlag.

Heinze, Franziska, und Frank König. 2019. Beobachtungen zur Interaktion zivilgesellschaftlicher und staatlicher Akteure im Handlungsfeld „Geschlechtliche Vielfalt und sexuelle Orientierung “. Tagung der DVPW-Sektion „Policy-Analyse und Verwaltungswissenschaft“, 19. und 20.09.2019. Speyer: Deutsche Universität für Verwaltungswissenschaften.

Hennis, Wilhelm. 1961. Verfassungsordnung und Verbandseinfluß: Bemerkungen zu ihrem Zusammenhang im politischen System der Bundesrepublik. Politische Vierteljahresschrift 2(1):23-35. 
Hustedt, Thurid. 2013. Ministerialverwaltung im Wandel: Struktur und Rolle der Leitungsbereiche im deutsch-dänischen Vergleich. Baden-Baden: Nomos.

Hustedt, Thurid, und Sylvia Veit. 2014. Forschungsperspektiven auf Regierungs-und Verwaltungskoordination: Koordinationsprobleme und Erklärungsfaktoren. der moderne staat 7(1):17-36.

Jochem, Sven, und Nico A. Siegel. 2003. Konzertierung, Verhandlungsdemokratie und wohlfahrtsstaatliche Reformpolitik. In Konzertierung, Verhandlungsdemokratie und Reformpolitik im Wohlfahrtsstaat, Hrsg. Sven Jochem, 7-32. Wiesbaden: Springer.

Kalagi, Sarah. 2014. Die Rolle von Anwaltskanzleien bei der Gesetzesvorbereitung in der Ministerialverwaltung: Ursachen und Probleme am Beispiel der Finanzmarktstabilisierungsgesetze. Zeitschrift für Parlamentsfragen 45(3):647-668.

Kohler-Koch, Beate. 2016. Deutsche Industrieverbände: Studie zur Heterogenität der Verbandswirklichkeit. Zeitschrift für Politikwissenschaft 26(Supp. 2):53-74.

Krick, Eva. 2010. Regieren mit Gipfeln - Expertengremien der großen Koalition. Zeitschrift für Politikwissenschaft 20(2):233-265.

Lehmbruch, Gerhard. 1987. Administrative Interessenvermittlung. In Verwaltung und ihre Umwelt, Hrsg. Adrienne Windhoff-Héretier, 11-43. Opladen: Westdeutscher Verlag.

Lehmbruch, Gerhard. 2003. Verhandlungsdemokratie: Beiträge zur vergleichenden Regierungslehre. Wiesbaden: VS.

Leif, Thomas, und Rudolf Speth. 2006. Die fünfte Gewalt: Lobbyismus in Deutschland. Bonn: Bundeszentrale für politische Bildung.

Loer, Kathrin, und Annette Töller. 2019. Interessenvertretung und politisches System in Deutschland im Wandel. Bonn: Bundeszentrale für Politische Bildung.

Loer, Kathrin, und Helmut Voelzkow. 2013. Moving away from corporatism. In Civil societies compared: Germany and the Netherlands, Hrsg. Annette Zimmer, 247-287. Baden-Baden: Nomos.

Lösche, Peter. 2007. Verbände und Lobbyismus in Deutschland. Stuttgart: Kohlhammer.

Mai, Manfred. 2016. Regieren in der modernen Gesellschaft: Governance aus der Sicht der Ministerialbürokratie. Leverkusen: Barbara Budrich.

Mai, Manfred. 2018. Interessenvertretung und Lobbyismus aus der Sicht der Ministerialbürokratie. Erfahrungen aus dem Bereich der Medienpolitik. Duisburg: Universität Duisburg, NRW School of Governance.

Mayer, Klaus, und Natalie Naji. 2000. Die Lobbyingaktivitäten der deutschen Wirtschaft. Recht und Politik 36(1):31-43.

Mayntz, Renate. 1978. Die Soziologie der öffentlichen Verwaltung. Heidelberg: C.F. Müller Verlag.

Mayntz, Renate, und Fritz W. Scharpf. 1975. Policy-making in the German federal bureaucracy. Amsterdam: Elsevier.

Müller, Edda. 1986. Innenwelt der Umweltpolitik. Sozial-liberale Umweltpolitik-(Ohn) macht durch Organisation. Opladen: Westdeutscher Verlag.

Müller, Edda. 2013. Exekutive und Legislative - an der Leine mächtiger Interessen? In Vom Hund und vom Schwanz. Zum Verhältnis von Exekutive und Legislative, Hrsg. Detlef Horster, 59-69. Veilerswist: Velbrück Wissenschaft.

Naggert, Matthias. 2018. Advocatus Politicus: Zum Wesen, den Motiven und Folgen anwaltlichen Engagements im politischen Raum. Baden-Baden: Nomos.

Niederhafner, Stefan, und Rudolf Speth. 2004. Die Ministerialbürokratie in Deutschland - Vom Kellner zum Koch. Forschungsjournal Neue Soziale Bewegungen 17(3):23-35.

Offe, Claus. 1981. The attribution of public status to interest groups: observations on the West German case. In Organizing interests in western Europe, Hrsg. Suzanne D. Berger, 123-158. Cambridge: Cambridge University Press.

Ossenbühl, Fritz. 2012. Outsourcing von Gesetzesentwürfen. Ein Scheinproblem. In Dynamik und Nachhaltigkeit des Öffentlichen Rechts - Festschrift für Professor Dr. Meinhard Schröder zum 70. Geburtstag, Hrsg. Matthias Ruffert, 359-370. Berlin: Duncker \& Humblot.

Paquet, Robert. 2009. Motor der Reform und Schaltzentrale: Die Rolle des Bundesministeriums für Gesundheit in der Gesundheitsreform 2007. In Gesundheitsreform 2007. Nach der Reform ist vor der Reform, Hrsg. Wolfgang Schroeder, Robert Paquet, 32-49. Wiesbaden: VS.

Pfeiffer, Ulrich, und Bernd Faller. 1997. Qualität des Verwaltungshandelns: zur Modernisierung der Bundesministerien; Gutachten. Bonn: Friedrich-Ebert-Stiftung.

Sack, Detlef, und Christoph Strünck. 2016. Zeitschrift für Politikwissenschaft 26, Sonderheft 2. Verbände unter Druck. Protest, Opposition und Spaltung in Interessenorganisationen.

Schiffers, Maximilian. 2019. Lobbyisten am runden Tisch: Einflussmuster in Koordinierungsgremien von Regierungen und Interessengruppen. Wiesbaden: Springer. 
Schmidt, Manfred G. 2011. Das politische System Deutschlands: Institutionen, Willensbildung und Politikfelder, 2. Aufl., Bonn: Bundeszentrale für politische Bildung.

Schmieszek, Hans-Peter. 2013. Großkanzleien als Ersatzgesetzgeber? Zeitschrift für Rechtspolitik 46(6): 175-178.

Sebaldt, Martin, und Alexander Straßner. 2004. Verbände in der Bundesrepublik Deutschland: Eine Einführung. Wiesbaden: VS.

Siefken, Sven T. 2007. Expertenkommissionen im politischen Prozess. Wiesbaden: VS.

Stefes, Christoph. 2014. Energiewende: Critical Junctures and Path Dependencies Since 1990. Zeitschrift für Politikwissenschaft Sonderheft 6:45-68.

Steinberg, Rudolf. 1979. Politik und Verwaltungsorganisation: zur Reform d. Regierungs-u. Verwaltungsorganisation unter bes. Berücks. d. obersten Bundesbehörden in d. Vereinigten Staaten von Amerika. Baden-Baden: Nomos.

Streeck, Wolfgang. 1994. Staat und Verbände: Neue Fragen. Neue Antworten? Politische Vierteljahresschrift: Sonderheft Staat und Verbände 25:7-36.

Streeck, Wolfgang. 2003. No longer the century of corporatism: Das Ende des Bündnisses für Arbeit. Köln: MPIfG working paper.

Süddeutsche Zeitung. 2016. Bundesrechnungshof: Bundesregierung verschleiert Beraterkosten

Tagesspiegel. 1999. In der Verbraucherszene herrscht dicke Luft.

Tageszeitung. 2013. Zahl der Einflüsterer in den Bundesministerien sinkt. 11. Okt. 2013.

Urban, Hans-Jürgen. 2010. Niedergang oder Comeback der Gewerkschaften? Aus Politik und Zeitgeschichte 57(13-14):3-7.

Veit, Sylvia, Nadin Fromm, und Falk Ebinger. 2018. „NEIN“ zu sagen ist eine unserer wichtigsten Pflichten. Politisierung, Rollenverständnis und Entscheidungsverhalten von leitenden Ministerialbeamt* innen in Deutschland. der moderne staat-dms 11(2):413-436.

Wambach, Manfred M. 1971. Verbändestaat und Parteienoligopol: Macht und Ohnmacht der Vertriebenenverbände. Stuttgart: Enke.

Weber, Jürgen. 1981. Interessengruppen im politischen System der Bundesrepublik Deutschland, 2. Aufl., München: Bayerische Landeszentrale für politische Bildungsarbeit.

Wehrmann, Iris. 2007. Lobbying in Deutschland - Begriff und Trends. In Lobbying: Strukturen. Akteure. Strategien, Hrsg. Ralf Kleinfeld, Annette Zimmer, und Ulrich Willems, 36-64. Wiesbaden: VS.

Weingart, Peter, und Justus Lentsch. 2008. Wissen-Beraten-Entscheiden. Form und Funktion wissenschaftlicher Politikberatung in Deutschland. Weilerswist: Velbrück Wissenschaft.

Wewer, Göttrik. 2015. Die Lobby des Guten - Interesse und Gemeinwohl im digitalen Zeitalter. Kiel: Lorenz-von-Stein-Institut.

Wirtschaftswoche 2019. „Spitze des Eisbergs“: Regierungsberater kosteten seit 2006 mehr als 1,2 Mrd. €. 2. Apr. 2019. 